\title{
REVIEWS
}

\section{Water at charged interfaces}

Grazia Gonella ${ }^{1,10}{ }^{10}$, Ellen H. G. Backus ${ }^{1,2}$, Yuki Nagata ${ }^{1}$, Douwe J. Bonthuis (1) ${ }^{3}$, Philip Loche (iD ${ }^{4}$, Alexander Schlaich $\mathbb{1}^{4,5}$, Roland R. Netz ${ }^{4}$, Angelika Kühnle ${ }^{6}$, Ian T. McCrum (1) ${ }^{7}$, Marc T. M. Koper ${ }^{7}$, Martin Wolf ${ }^{8}$, Bernd Winter ${ }^{8}$, Gerard Meijer (1) ${ }^{8}$, R. Kramer Campen ${ }^{8,9}$ and Mischa Bonn (1) ${ }^{1 凶}$

Abstract $\mid$ The ubiquity of aqueous solutions in contact with charged surfaces and the realization that the molecular-level details of water-surface interactions often determine interfacial functions and properties relevant in many natural processes have led to intensive research. Even so, many open questions remain regarding the molecular picture of the interfacial organization and preferential alignment of water molecules, as well as the structure of water molecules and ion distributions at different charged interfaces. While water, solutes and charge are present in each of these systems, the substrate can range from living tissues to metals. This diversity in substrates has led to different communities considering each of these types of aqueous interface. In this Review, by considering water in contact with metals, oxides and biomembranes, we show the essential similarity of these disparate systems. While in each case the classical mean-field theories can explain many macroscopic and mesoscopic observations, it soon becomes apparent that such theories fail to explain phenomena for which molecular properties are relevant, such as interfacial chemical conversion. We highlight the current knowledge and limitations in our understanding and end with a view towards future opportunities in the field.

Water in contact with charged interfaces is relevant to a plethora of geological, atmospheric and biological processes, as well as technological applications such as in drug design, bioimplants, energy production and storage devices. In the environment, water is characteristically in contact with minerals, the surfaces of which are typically charged because rarely does the $\mathrm{pH}$ of the aqueous solution coincide with the surface's point of zero charge, also known as the isoelectric point. Mineral dissolution and growth can alter the surface charge of the mineral itself and the ion composition of the surrounding aqueous solution, with an effect on the chemistry of aqueous systems, such as oceans $s^{1-4}$. Furthermore, ocean acidification caused by the dissolution of excess carbon dioxide from the atmosphere $\left(\mathrm{CO}_{2}+\mathrm{H}_{2} \mathrm{O} \rightleftharpoons\right.$ $\left.\mathrm{H}_{2} \mathrm{CO}_{3} \rightleftharpoons \mathrm{HCO}_{3}{ }^{-}+\mathrm{H}^{+} \rightleftharpoons \mathrm{CO}_{3}{ }^{2-}+2 \mathrm{H}^{+}\right)$impacts marine life, because acidic conditions affect the stability of biominerals, such as the shells of sea animals. In living matter, water is in contact with charged cell membranes that separate organelles and the cytoplasm from the environment. Ions and other small molecules (osmolytes) are dissolved in the aqueous physiological environment. The membrane charges, water structure and ion interactions affect the structure, stability, dynamics and function of the biomembranes ${ }^{5-7}$. An important role of interfacial water at biomembranes is to assist the in-plane proton conductivity along the membrane that is vital for cellular bioenergetics. Experimental evidence shows that the surface protons do not rapidly equilibrate with bulk: a kinetic barrier exists for the flow of protons from the interfacial water layer on the outside of the cell into the surrounding bulk solution. However, the origin of this barrier, including the molecular-scale details of interfacial water that ensure that the interfacial proton has a strongly anisotropic diffusion coefficient, remains poorly understood ${ }^{8-15}$. Interfaces that involve charged surfaces and aqueous electrolytes are common in electrochemical measurements, as well as in reactions that produce fuel (for example, water splitting) or consume it (for example, $\mathrm{H}_{2}$ fuel cells). For instance, in oxide-based photocatalytic water splitting, $\mathrm{H}_{2}$ is produced from $\mathrm{H}_{2} \mathrm{O}$ on the surface of a metal oxide electrode on irradiation, and the photocatalytic activity is strongly affected by surface speciation and solution $\mathrm{pH}$, which, in turn, affect the surface charge ${ }^{16-20}$. In hydrogen fuel cells and electrolysers, hydrogen oxidation and evolution reactions take place at the electrode-water interface ${ }^{21-24}$. Electrochemical energy conversion efficiency is governed by the charge transfer at the boundary between the metal and the aqueous electrolyte solution ${ }^{25-28}$.

The systems described above have been chosen as representative examples to discuss water at charged interfaces and because they are systems that the authors have extensively investigated, but by no means are the 
only possible examples of charged interfaces ${ }^{6,17,29-35}$. All the systems considered in this Review feature a charged interface in contact with an aqueous solution containing ions. Based on the chemistry of the interface, the systems can be divided into three categories: (1) inorganic compounds (more generally, insulators or semiconductors either found as minerals in nature or in devices), characterized by a mechanically stiff interface, with localized surface charges; (2) biomembranes or (bio)molecules that have a deformable interface with localized charges; and (3) metals in which the charge is delocalized (for example, metallic electrodes). The interplay between mechanical properties and charge localization exists for a variety of interfaces involving planar surfaces, nanoparticles, droplets, liposomes and biological cells, independently of their surface curvature. While several recent reviews have examined the behaviour of water in one of these systems separately ${ }^{8,29,30,32-34,36-41}$, by considering them together, we aim to develop a more unified framework for water at charged interfaces, highlighting the current knowledge and limitations in our understanding. Such a unified framework requires understanding elementary processes as well as the characteristic length scales and timescales that span more than ten orders of magnitude. The multiscale nature of this problem furnishes acute challenges for both experiments and theory, highlighting the need for future method development.

We start by providing a historical overview of the classical mean-field models used to describe charged surfaces in contact with an aqueous electrolyte solution and then continue by detailing the different components of the system: the charged interface, water and ions. While generally offering a qualitatively accurate description of a variety of well-defined systems, mean-field models fail to offer a quantitatively accurate description of phenomena of interest for common systems, especially at the molecular level. Much recent experimental, theoretical and computational work highlights that many of these limitations result from, often implicit, assumptions about the structure of interfacial water and ions on which these models are based. However, a systematic addition of structural details allows an increasing breadth of systems that can be successfully described. For complex systems, the description of equilibrium properties of many aqueous solution-charged interfaces becomes challenging, and describing such systems under non-equilibrium conditions makes their

\footnotetext{
Author addresses

${ }^{1}$ Max Planck Institute for Polymer Research, Mainz, Germany.

2Department of Physical Chemistry, University of Vienna, Vienna, Austria.

${ }^{3}$ Institute of Theoretical and Computational Physics, Graz University of Technology, Graz, Austria.

${ }^{4}$ Physics Department, Free University of Berlin, Berlin, Germany.

5Université Grenoble Alpes, CNRS, LIPhy, Grenoble, France.

${ }^{6}$ Faculty of Chemistry, Bielefeld University, Bielefeld, Germany.

'Leiden Institute of Chemistry, Leiden University, Leiden, Netherlands.

${ }^{8}$ Fritz Haber Institute of the Max Planck Society, Berlin, Germany.

${ }^{9}$ Faculty of Physics, University of Duisburg-Essen, Duisburg, Germany.

${ }^{10}$ Present address: Institute of Biochemistry, Department of Biology, ETH Zürich,

Zürich, Switzerland.
}

description even more challenging. An example of such systems is presented in the next section, where we consider cases in which interfacial reactivity plays an important role. In these cases, the complexity is such that a full molecular description, including electronic structure, is often necessary to describe the observed phenomena. We end with a look at the future, discussing the modern challenges and the tools that offer the possibilities of tackling them.

\section{Classical mean-field description of charged interfaces}

The electrostatic attraction between a charged interface and counterions draws oppositely charged ions from the solution to the interface, competing with thermal effects that favour a more homogeneous, that is, bulk-like, ion distribution. The result is a charge distribution that is spatially inhomogeneous along the surface normal, with an associated electric field. This field acts on the static dipole of water, causing its preferential orientation near the interface. The interfacial ion distribution, together with the water molecules, form the electrical double layer (EDL), schematically shown in FIG. 1.

The classical EDL description dates to the late nineteenth to early twentieth century ${ }^{42-50}$ (FIGS 1,2). The first to propose a model to describe the EDL was Helmholtz, who treated the EDL as a parallel-plate capacitor: one plate corresponding to the highly charged interface and the other corresponding to the centre of the closest-approaching hydrated counterions (the outer Helmholtz plane, OHP). Together, these two plates define the Helmholtz layer (also called the Stern layer), across which the potential decreases linearly ${ }^{42,43}$. As pointed out later on by Grahame, Helmholtz's description of the EDL does not consider the presence of specifically adsorbed but not fully solvated ions. In Grahame's refinement, these ions are said to form the so-called inner Helmholtz plane (IHP) ${ }^{48}$. The model by Gouy and Chapman ${ }^{44,45}$ was proposed to describe moderately charged surfaces in contact with aqueous electrolyte solutions by using the Poisson-Boltzmann equation to describe the equilibrium distribution of ions. This results in an almost exponential decay of the potential, moving away from the surface. This mean-field description assumes that (1) the surface is laterally homogeneous and charge distributions can be described by their spatial average in the plane of the interface, meaning that there are no domains or charged patches; (2) the charged surface is perfectly sharp; (3) ions are point charges and only interact through Coulombic interactions; (4) there are no ion-ion correlations; and (5) water is a homogeneous dielectric continuum. The Gouy-Chapman model accounts for many experimental observations, mostly because electrostatic interactions are long-ranged and the vast majority of local molecular-level details are effectively neglected. The Debye-Hückel approximation ${ }^{46}$ results from a linearization of the nonlinear Poisson-Boltzmann equation and returns an exponentially decaying potential. Although strictly speaking the Debye-Hückel theory is limited to low potentials (low charge), it often works remarkably well also for nominally highly charged surfaces, because 


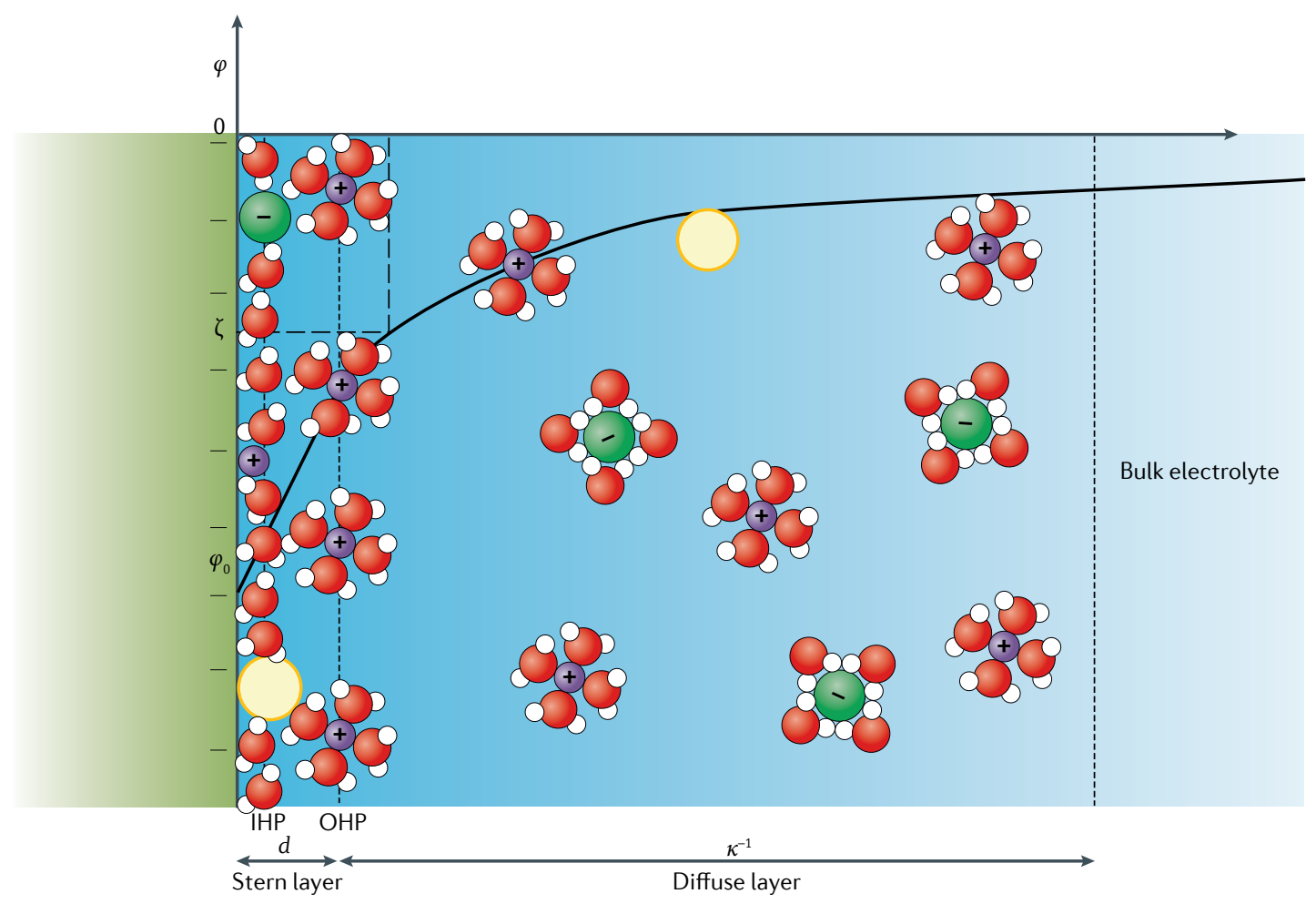

Fig. 1 | Classical mean-field description of the electrical double layer. Schematic description of the Gouy-ChapmanStern model. Indicated are the inner Helmholtz plane (IHP), the outer Helmholtz plane (OHP), the thickness of the Stern layer, $d$, and the thickness of the diffuse layer, also known as the Debye length, $\kappa^{-1}$. Also indicated is the potential $\varphi$ as a function of the distance from the surface. The Debye length is assumed here to be substantially larger than the other length scales (atom sizes, water molecules and interface), as appropriate for low salt concentration. For high salt concentrations, all length scales are comparable, making the theoretical description of charged surfaces even more difficult. The purple, green and yellow spheres represent the cations, the anions and neutral species, respectively. The hydration water around the ions as well as at the surface are explicitly represented. The definition of the surface potential, $\varphi_{0}$, can be found in BOX 1 , while an in-depth discussion on how to measure the potential at the shear plane, $\zeta$-potential, can be found in BOX 2 .

counterions condense near the surface and, thereby, decrease (or renormalize) the effective surface charge. On a side note, although these models have been discussed for planar surfaces, they similarly apply to curved surfaces if the radius of curvature is larger than the Debye screening length, $\kappa^{-1}$,which is the characteristic length associated with the potential decay into the bulk ${ }^{51}$.

The shortcomings of the Gouy-Chapman model have been the focus of theoretical investigations for decades $^{52}$. One such major shortcoming is the assumption that ions are point charges that, in high fields, can approach the interface at an infinitesimally small distance. This approximation is unrealistic because of the ions' finite size, their hydration shell and possible surface solvation effects ${ }^{53}$. The Stern model $^{47}$ introduces finite-sized ions by combining the Helmholtz $z^{42,43}$ and Gouy-Chapman ${ }^{44,45}$ models and accounts for a reduced interfacial water dielectric constant. In this model, one finds the Gouy-Chapman diffuse layer beyond the OHP. The Stern model is often called the Gouy-ChapmanStern (GCS) model and is shown in FIG. 1. The arrangement of ions in the solution near the interface screens the surface charges and, thus, decreases the local potential, and its Debye length, $\mathcal{K}^{-1}$ (FIG. 1), can be defined as follows:

$$
\kappa^{-1}=\sqrt{\left(\varepsilon_{0} \varepsilon_{\mathrm{r}} k_{\mathrm{B}} T / \sum_{j=1}^{N} n_{j}^{0} q_{j}^{2}\right)}
$$

where $\varepsilon_{0}, \varepsilon_{\mathrm{r}}, k_{\mathrm{B}}$ and $T$ are the vacuum permittivity, relative static permittivity, Boltzmann constant and absolute temperature, respectively. $n_{j}^{0}$ and $q_{j}$ are the bulk charge density and the charge of species $j$, respectively, and $N$ is the total number of charged species. The Debye length decreases with increasing concentration of ions in the solution: for biomembranes, under physiological conditions, the Debye length is $\sim 1 \mathrm{~nm}$, whereas for a charged mineral at circumneutral $\mathrm{pH}$ and very low salt concentrations, it can be as large as $1 \mu \mathrm{m}$. Bockris et al. further enriched the description of the EDL by accounting for the dipolar nature of the water molecules. Thus, they proposed a refinement of the model in which the permittivity of water assumes three different values: one for the diffuse layer (coinciding with that of the bulk solution), a smaller one for the first layer of molecules next to the charged surface (as, here, water is possibly depleted and its dipole unable to rotate - dielectric saturation) and a third, in between the other two, for the second layer of water next to the surface ${ }^{49,50,54,55}$. 


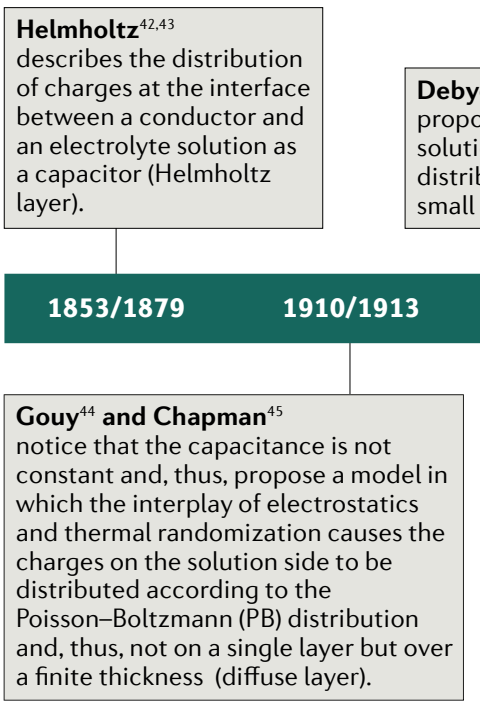

Grahame ${ }^{48}$

proposes that some ionic (losing at least partially their hydration shell) or uncharged species penetrate the Helmholtz layer and specifically adsorb to the surface. Thus, further separating the Stern layer in inner Helmholtz (locus of the centres of the specifically adsorbed ions) and outer Helmholtz layers (locus of the centres of the non-specifically adsorbed hydrated ions).

Fig. 2 | Timeline. Milestone developments of the classical mean-field description of the electrical double layer.

In the past few decades, substantial effort has been devoted to characterizing the behaviour of charged interfaces in contact with electrolyte solutions to test the aforementioned classical models. In many cases, these classical models provided an adequate description, but, in many more, the models were either quantitatively off or downright qualitatively inadequate. Some of such historical works that used the GCS model are, for instance, those by Grahame, who measured the differential capacitance of $\mathrm{Hg}$ in contact with an aqueous $\mathrm{NaF}$ solution ${ }^{48}$, that by McLaughlin and colleagues, who measured the potential at the shear plane (close to the OHP, also known as the $\zeta$-potential - see BOXES 1,2) of liposomes of mixed composition (zwitterionic and charged phospholipids) ${ }^{56}$, and by Israelachvili and Adams, who measured the double-layer forces (BOX 3) between mica surfaces in $\mathrm{KNO}_{3}\left(\mathrm{REF}^{57}\right)$. The shortcomings of these models typically became clear when changing some experimental conditions. For Grahame's work, this happened when increasing the applied potential or the solution's ionic strength ${ }^{48}$, for McLaughlin and colleagues when, instead of a singly charged lipid, a triple negatively charged one was used ${ }^{58}$, and for Israelachvili and Adams, the models failed for concentrated solutions or in close proximity of the charged surface ${ }^{57}$. Thus, while successfully describing some phenomena occurring on mesoscopic and macroscopic length scales, the classical GCS mean-field theory often fails to describe situations of interest. As we describe in this Review, this failure is often expected: it turns out that many systems of great practical interest violate the assumed behaviour of water and ions in such treatments. For example, much experiment and theory demonstrates that, to describe electron transfer across (charged) solid-water interfaces, such as for the photocatalytic water splitting into hydrogen and oxygen at an electrode-solution interface, details of the interfacial molecular arrangement of water are quintessential. The interface can act to align water molecules, but the water molecules can act back on the interface, through both protonation and deprotonation, and dissociative adsorption at the interface ${ }^{59,60}$, thus changing the local charge and surface potential (FIG. 1; BOX 1). The interplay between the interface, its charges, water molecules and ions makes the charged interface-aqueous solution more than the sum of its parts, highlighting the importance of the molecular details and the inadequacy of the description of water as a homogeneous dielectric medium assumed in traditional mean-field theories. In the following, while describing the nature of the interfacial charge, water structure and ions in more detail, this classical description's limitations will become clear.

\section{Origin and nature of the surface charge}

The origin of the charge at a surface in contact with water can be traced to five main sources: (1) the application of an externally controlled potential as in electrochemistry (FIG. 3a); (2) adsorption of ions from or dissolution of ions into the bulk (FICS 1,3c); (3) protonation and deprotonation of surface groups (FIG. 3b); (4) ion exchange between solution and surface (FIG. $3 \mathrm{C}$ ); and (5) hydrolysis of surface groups (followed by protonation or deprotonation) (FIC. 3d).

Electrically conducting electrode-electrolyte interfaces are unique in that the electrical connection between the electrode and an external circuit allows a continuous transfer of charges to and from the electrode-electrolyte interface, even at a constant applied potential. Besides the fact that this charge is delocalized, the potential at the interface can be controlled externally (while the systems we describe below have a surface potential determined by the solid surface structure and the solution composition). In mechanism (1), the potential at which the electrode surface is uncharged (the potential of zero charge, $E_{\mathrm{pzc}}$ ) depends on the chemical nature and surface structure of the electrode, as well as the nature of the electrolyte. It is expected that at the $E_{\mathrm{pzc}}$, the electric field across the surface is vanishing, except for a dipolar contribution resulting from the specific interaction of the water with the electrode. If at the electrode-electrolyte interface no charge-transfer reactions occur, the surface 
charge density can also be tuned. As the potential is varied, the negative or positive surface charge density of the electrode changes and the electric field can change sign, affecting water orientation and, possibly, the chemistry of the electrode-electrolyte interface.

Mechanism (2) for the formation of surface charge, for example, counterion dissolution, is characteristic of ionic surfactants (FIG. 3b) and ionic crystals (FIG. 3c). Ionic surfactants are charged, amphiphilic, surface-active molecules, the counterions of which become hydrated and can move away from the surfactant into the bulk, when in contact with liquid water. In case the counterion is a proton (mechanism (3)), the $\mathrm{pH}$ of the aqueous solution determines whether the proton goes into solution or not. For biomembranes, the $\mathrm{pH}$ of the aqueous solution seldom coincides with the $\mathrm{pH}$ value for which the surface is uncharged, that is, the point of zero charge. For instance, it is generally assumed that physiologically relevant $\mathrm{pHs}$ are slightly alkaline, $\sim 7.4$; however, wide deviations from this value are often observed in living systems. In solid tumours, the extracellular $\mathrm{pH}$ is slightly acidic, with a value of $\sim 6.7$ (REFS ${ }^{61,62}$ ) and, even in healthy cells, the $\mathrm{pH}$ of the organelles of the secretory and endocytic pathways ranges from 4.7 to 6.7 , while that of the mitochondrial matrix is $\sim 8.0\left(\mathrm{REF}^{63}\right)$. These acidic or basic conditions can favour protonation or deprotonation of the lipid headgroups, thus affecting the net charge of the membrane. A typical example is the membrane of mammalian cells that, under physiological conditions, is negatively charged mainly because the positive counterions of negatively charged lipid headgroups go into solution ${ }^{64,65}$. Biological membranes are characterized by nominal surface charge densities ranging from -0.002 to $-0.3 \mathrm{C} \mathrm{m}^{-2}\left(\mathrm{REF}^{31}\right)$. In these membranes, also lipids with zwitterionic headgroups are present, which do not contribute to the net charge of the surface, even though they favour anion adsorption ${ }^{66}$, but do create a dipolar field that contributes to the alignment of water molecules ${ }^{67}$ (as detailed below). The picture is further complicated because the local concentration of hydrated protons at the interface can be quite different from that in the bulk ${ }^{68,69}$ and because different ions can affect the $\mathrm{p} K_{\mathrm{a}}$ values differently (for example, through ion-specific effects, discussed in the next section $)^{64,70-73}$.

Similarly, the surface of ionic crystals (mechanism (4)) can become charged because one ionic component

\section{Box 1 | The surface potential: definition and its experimental determination}

The surface potential, $\varphi_{0}$ (FIG. 1), is defined by the difference in electrical potential between that at the surface and infinity, which, in practical terms, means the solution bulk. As usually the potential at infinity is set to 0 , this difference in potential and $\varphi_{0}$ coincide. This deceptively simple definition turns out to be more complex in reality: the surface is not infinitely thin or sharp, and one has to define the potential at a certain position. The finiteness of such a boundary region has led to the introduction of the concept of Gibbs dividing surface, defined as the plane where the surface excess equals zero, that is, where the density profile reaches the average value between that of the two bulk phases. Still, this is a 'continuum model' definition of a surface because the local and temporal heterogeneities of real surfaces are smoothened out. The definition of $\varphi_{0}$ turns out to be not so trivial, nor is its experimental determination. It is still debated whether it can be measured at all and, if so, whether it refers to the potential at the surface on the solid or on the solution side ${ }^{246}$. Different methods have been used to measure $\phi_{0}$, but, in BOX 2, we focus on some of the most common. dissolves in water more readily than the other, leaving an unbalanced charge at the surface (FIG. 3c). One example is $\mathrm{CaF}_{2}$ at acidic $\mathrm{pH}$, in which the ready dissolution of fluoride ions prompts a positively charged surface ${ }^{74,75}$. Moreover, a possible (intrinsic) source of charge can result from ion substitution in the lattice during crystallization. Hydrolysis at the surface (mechanism (5)) of oxides is another possible source of charge, as with silica, where the reaction with water generates silanol ( $\mathrm{Si}-\mathrm{OH}$ ) termination of the surface ${ }^{76}$ (FIG. $3 \mathrm{~d}$ ). These silanols, depending on the $\mathrm{pH}$ of the aqueous solution, can be deprotonated, leading to a negatively charged silica surface 77,78 . Another common phenomenon, especially in clay minerals, is a structural charge generated from ion exchange (mostly cation) in the lattice ${ }^{79}$.

The classical mean-field description relies on the concept of average charge density, which is appropriate for ideal metals. However, mean-field theories cannot capture the interaction of water with localized charges present at both mineral interfaces and biointerfaces. Even so, lacking a better theory, many of the observed behaviours in these systems ${ }^{80-84}$ have been described using mean-field theories. For example, one of the phenomena that cannot be tackled by mean-field descriptions is the intriguing surface charge regulation of proteins by charge-neutral biological osmolytes - small molecules, such as urea and glycerol, that are synthesized by organisms ${ }^{85}$. This phenomenon has been experimentally investigated by using silica to mimic the behaviour of membrane proteins, because the $\mathrm{p} K_{\mathrm{a}}$ of the silanol groups on its surface is close to that of two abundant amino acids, namely, aspartic and glutamic acid. It was observed that urea accumulates at the silica-water interface, resulting in an increase in the silica charge by $\sim 50 \%$ (equivalent to an increase of $4 \mathrm{pH}$ units), whereas glycerol was depleted from the interface, resulting in neutralization of the surface charge (equivalent to reducing the $\mathrm{pH}$ by 2 units ${ }^{86}$. A discussion on overcharging and charge-inversion effects by charged species is presented in the next section.

We should also keep in mind that most surfaces are not only spatially heterogeneous but also highly dynamic (temporally heterogeneous), a property that considerably complicates their molecular-level description. The spatial heterogeneity is nicely exemplified in biomembranes, which exhibit a soft surface with charge-bearing constituents that are laterally mobile ${ }^{87}$ : a time-dependent, non-uniform charge distribution ${ }^{88}$. Another example is the charged silica-water interface, for which the macroscopic wetting angle results from the subtle interplay between nanometre-scale hydrophobic and hydrophilic (potentially charged) patches on the surface ${ }^{89}$. For silica, the continuous protonation and deprotonation of surface oxide groups - temporal heterogeneity - leads to a constant average charge but strongly varying charge effects visible on the micrometre scale ${ }^{90}$. Interestingly, in all three works cited above, these spatiotemporal heterogeneities in surface charge have been probed through the effect they induce on water orientation. This fact further highlights the mutual dependence between the charged surface and water behaviours, the leading theme of this Review. The few selected examples discussed above are 


\section{Box 2 | Experimental determination of surface potential}

Electrokinetic methods. In colloidal science, information on particle charge is typically derived by electrophoretic measurements, used to look at the mobility of these particles in response to an externally applied electric field. Depending on the concentration of ions in solution, different equations can be used to obtain what is called the $\zeta$-potential, defined as the potential at the shear plane (FIG. 1). While exact models exist for the limiting cases of low and high salt concentrations (or, equivalently, small and big spherical particles), only approximations are available at intermediate concentration ${ }^{247}$. In any case, this deduced $\zeta$-potential is the potential at the shear plane, which includes the immobile layer formed around the particles that is moving along with the particles under an external electrical field. As such, the $\zeta$-potential is smaller than $\varphi_{0}$ because of the counterions contained in the immobile layer at the particle surface. Note that the shear plane is close but not identical to the outer Helmholtz plane (OHP, see FIG. 1). For planar surfaces, the equivalent of a colloidal $\zeta$-potential measurement is a streaming measurement. In this case, the $\zeta$-potential of the surface is obtained using a capillary channel made of the material of interest and by applying an external pressure to induce the liquid to flow from one side of the capillary to the other. The equilibrium electrical double layer (EDL) formed in the electrolyte solution in response to the material surface charge is then disrupted by the liquid flow and the counterions creating an excess of counterions downstream and an excess of co-ions upstream. This asymmetry in charge distribution induced by the applied mechanical pressure induces a potential difference between the inlet and the outlet, which reflects the $\zeta$-potential.

Vibrating plate methods. The vibrating capacitor method is widely used for planar surfaces. Its invention dates back to Lord Kelvin and measures a capacitance developed in the non-conducting gap between the material of interest and a metal electrode. When the electrode vibrates, an AC current develops in the circuit as a result of the potential difference between the two media. A DC voltage is then applied to compensate for such current. For lipid monolayers at the air-water interface, one electrode is above the monolayer, while the other electrode is immersed in water below the monolayer ${ }^{248-252}$. With this method, one measures the difference in potential between the air-water and the air-lipid-water interface. This potential difference depends on all the group dipoles in the lipid molecule and the water molecules in the EDL influenced by the surface potential ${ }^{248,253}$. The dipole potential appears to be the principal contributor and is dominated by the carbonyl dipole ${ }^{254}$.

Kelvin probe microscopy. For solid planar surfaces, the Kelvin method has been combined with an atomic force microscopy tip to obtain Kelvin probe force microscopy, which allows to measure the local contact potential difference between the oscillating tip and the sample, thereby mapping the surface potential (work function) of the sample with high spatial resolution ${ }^{255,256}$. This local potential is the contact potential difference between the two materials and is directly related to the difference in their work functions. It can be related to the local charge density weighted by a tip-shape-dependent weight function ${ }^{241,257,258}$. Recently, Kelvin probe force microscopy has been applied to interfaces involving electrolyte solutions ${ }^{259}$.
Force-distance methods. These methods are used to measure the force between two surfaces, such as atomic force microscopy ${ }^{97}$ and surface force apparatus (BOX 3). From the measured force, a value for the potential can be derived. In this derivation, an exponential dependence of the potential on the distance of the kind $e^{-\kappa x}$ is assumed, where $\kappa^{-1}$ is the Debye length and $x$ the distance from the OHP (FIG. 1). By extrapolating in the limit $x \rightarrow 0$, a value for the potential at the OHP can be obtained.

Electron spectroscopy methods. Photoelectron spectroscopy, and X-ray spectroscopy in particular, rely on the emission of core electrons following the adsorption of a photon (BOX 4). The application of X-ray spectroscopy techniques to solid and gas phases has a long history ${ }^{36}$, but its use for volatile aqueous phases has been established recently with the introduction of the vacuum liquid microjet technique ${ }^{261,262}$, enabling the acquisition of photoemission spectra from thin aqueous solution films in a millibar pressure gas environment. An alternative method that circumvents the exposure of the liquid phase to vacuum uses liquid cells equipped with a few-nanometre-thick membrane to separate the liquid from vacuum ${ }^{17,263-265}$. The membrane is thin enough to transmit the emitted photoelectrons, thus enabling their detection. These developments have tremendously expanded the scope of photoemission spectroscopy, which has been recently used to determine both the EDL profile at a polycrystalline gold electrode ${ }^{91}$ and the surface potential of $\mathrm{SiO}_{2}$ nanoparticles ${ }^{145}$. The elegance of this approach is that $\varphi_{0}$ can be directly inferred from the measured binding energy of a core-level electron in a nanoparticle, as the photoelectron loses or gains kinetic energy in direct proportion with the potential as it travels from the surface to the detector.

\section{Nonlinear optical spectroscopy methods. Nonlinear optical} spectroscopies such as sum-frequency generation (SFG) spectroscopy and second-harmonic generation (SHG) are inherently surface-sensitive because of the breaking of the symmetry at the interface between different media (BOX 5). When charges are present at the interface, these charges effectively break the symmetry, either by the field-induced alignment of dipolar molecules or by the field-induced polarization of the nearby liquid. So the SFG and SHG intensities are typically assumed to be proportional to $\left(\varphi_{0}\right)^{2}\left(\mathrm{REFS}^{80,82,266-268}\right)$. In case water is the only contributor to the second-order signal, non-resonant SHG has been used as a direct reporter of the surface potential ${ }^{80,267,269,270}$. When, however, other components in the system exhibit a net orientation, these can also contribute to the signal, as is, for instance, the case of lipid monolayers at the air-water interface ${ }^{254}$. Non-resonant SHG has been used to learn about the surface potential and charge of mineral surfaces as well as membranes $^{80,157,271-273}$, has been refined using heterodyne detection ${ }^{268}$ and has been shown, in scattering geometry, to provide information on $\varphi_{0}$ from the surface of particles, droplets and vesicles ${ }^{267,274,275}$. Using SHG microscopy, the aligned water molecules have been used as reporters of surface heterogeneity and dynamics at mineral interfaces ${ }^{90}$, asymmetrical lipid bilayer membranes ${ }^{88}$ and neuronal membranes ${ }^{276}$. We note that Gouy-Chapman and Stern models are required to obtain a value of this surface potential from SHG or SFG intensity. not amenable to be described by a mean-field model of the charged interface (which assumes lateral homogeneity and sharp discontinuity across the interface) and highlight the deficiencies of such a model that necessarily cannot account for non-ideal interfacial systems and complex phenomena.

\section{The role of ions}

The spatial distribution of ions at a charged interface plays a crucial role in determining the effect of charge on the water organization. In mean-field theories, the surface charge is screened by the counterions, and the degree of screening, that is, the decrease in potential away from the surface, is determined by the bulk ion concentration. A combined ambient-pressure X-ray photoelectron spectroscopy (BOX 4) and computational study investigated the potential drop in the electrolyte solution (FIC. 1; BOX 1) and, by using a neutral spectator molecule, unveiled the shape of the EDL profile both as a function of the applied bias and electrolyte concentration ${ }^{91}$. However, the signal-to-noise ratio of these experiments did not allow to discern whether the Gouy-Chapman or the GCS description was more appropriate. Interestingly, marked deviations of classical mean-field theory from 
experiments have been reported for the screening efficiency. When measuring the normal force between two crossed cylindrical mica surfaces (BOX 3) in concentrated electrolyte solutions of various nature (aqueous $\mathrm{NaCl}$,

\begin{abstract}
Box 3 | Surface force apparatus and scanning probe microscopy
The surface force apparatus (SFA) has been developed to directly measure forces between two atomically smooth surfaces in a defined atmosphere or in liquids. Briefly, the distance between two cylinders (see the figure, part a) in crossed geometry is controlled within $\sim 0.1 \mathrm{~nm}$. The force acting between the two surfaces is obtained by measuring the distance (typically, optical interferometry) and the stiffness of the spring supporting one of the cylinders. This provides a force resolution of $\sim 10^{-7}-10^{-8} \mathrm{~N}$. SFA is very valuable to elucidate fundamental interactions between surfaces in general and electrical double layer forces in particular ${ }^{277}$.

While SFA provides high spatial resolution along the surface normal direction, its lateral resolution is usually low $(\sim 1 \mu \mathrm{m})$. Scanning probe microscopy (SPM) techniques overcome this drawback ${ }^{278}$. SPM offers the capability to directly image a surface with atomic resolution, providing unparalleled insights into the atomic structure of surfaces. In a scanning tunnelling microscope (STM), a sharp tip is scanned at close distance (several $\AA$ ) over the surface of interest (see the figure, part b). Owing to the tunnelling effect, an electric current flows between the tip and an electrically conducting sample. As the tunnelling current depends on both the distance between the tip and the surface and the electronic structure of the materials, it reveals local surface information. It is worth noting that an STM can also be operated in an electrolyte solution when the tip is coated with an insulating layer, except for the very end of the tip. To overcome the limitation to conducting materials, atomic force microscopy (AFM) can be used to detect the force acting between the tip and the sample. When operated in dynamic mode, a force resolution down to $10^{-11} \mathrm{~N}$ can be achieved at a sub- $\AA$ s spatial resolution ${ }^{279}$. Recently, AFM imaging at the solid-liquid interface has been extended to map a volume above the surface ${ }^{195}$, providing high-resolution spatial information of the interfacial solvation structure.
\end{abstract}

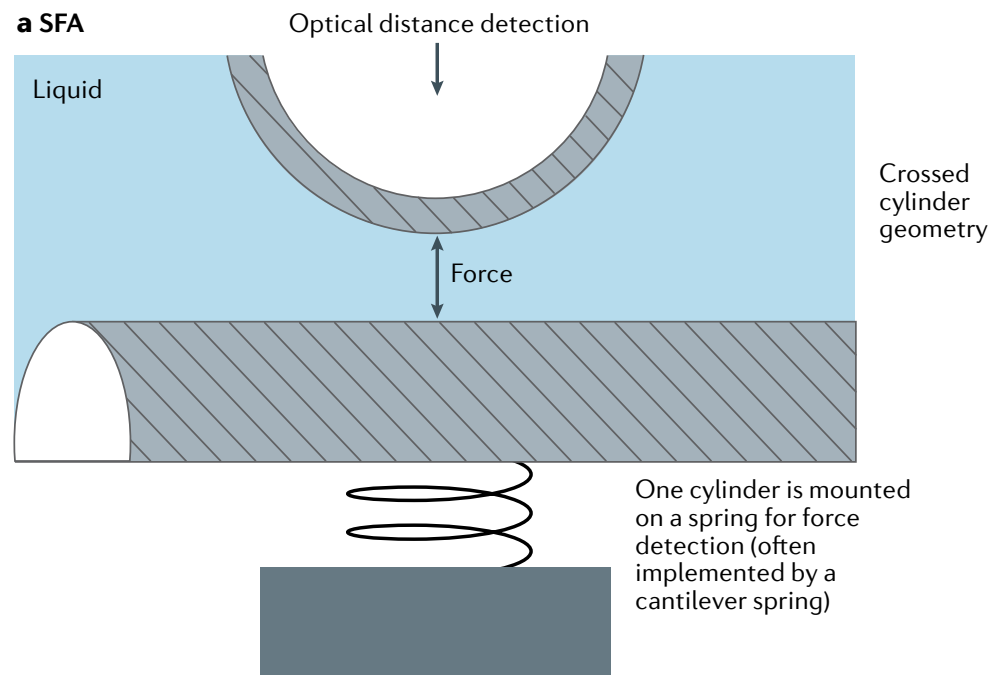

b SPM

Optical distance detection in AFM Tunnelling current measurement in STM

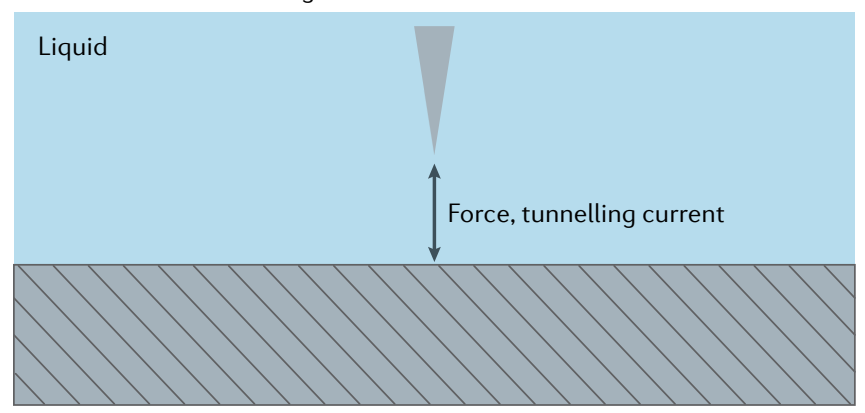

ionic liquids and ionic liquids diluted with propylene carbonate), the screening length has been observed to increase with concentration (underscreening) , $^{92}$, rather than decrease as predicted by mean-field theories. The opposite behaviour has recently been reported for flat $\mathrm{Pt}(111)$-aqueous $\mathrm{HClO}_{4}$ or $\mathrm{HF}(\mathrm{pH}$ 4) solution at low electrolyte concentration (ionic strength $<5 \times 10^{-3} \mathrm{M}$ ) by adding $\mathrm{NaClO}_{4}$ or $\mathrm{NaF}$, respectively. $\mathrm{Au}(111)$-aqueous $\mathrm{HClO}_{4}\left(\mathrm{pH} 3\right.$ ) solution with added $\mathrm{NaClO}_{4}$ (ionic strength $<10^{-1} \mathrm{M}$ ) also showed the same behaviour. In all these cases, the electrostatic screening of the surface charge is appreciably more efficient than that predicted by mean-field theories (overscreening) ${ }^{93}$.

Dating back at least to Hofmeister, with his observation of the ion-type-dependent ability to precipitate proteins, it has been known that ions are far from equal, even when they present the same valency ${ }^{94-105}$. Further, it has been shown that specific ion effects in lipid membranes also depend on the membrane composition and charge ${ }^{106-113}$, highlighting factors such as membrane compressibility and headgroup mobility, which, in turn, are intimately connected to the local hydrogen bond network. Ion-specific effects have also been demonstrated to be important for hard surfaces, such as, for instance, for silica ${ }^{97,114-120}$ or electrochemical systems ${ }^{121-126}$.

For biomembranes, it is also known that the ionheadgroup interaction appears in most cases not to be dominated by electrostatics ${ }^{70}$ but, rather, guided by matching water affinities ${ }^{127,128}$. When an ion is more strongly hydrated than its counterion, dehydration of the former requires more energy than the energy gained by forming the contact ion pair, thus favouring contact ion pairs of similarly hydrated ions ${ }^{127}$. Therefore, according to this principle, small cations (such as $\mathrm{Na}^{+}$) prefer small anions or anionic groups (such as acetate, $\mathrm{C}_{2} \mathrm{H}_{3} \mathrm{O}_{2}^{-}$), whereas larger cations (such as $\mathrm{K}^{+}$) are preferentially attracted to larger anions or anionic groups (such as methylsulfate, $\left.\mathrm{CH}_{3} \mathrm{OSO}_{3}^{-}\right)^{55}$.

Overcharging, or charge inversion, is the phenomenon observed when counterions from the solution adsorb on the surface, forming the Stern layer, in such a way that their total charge in magnitude is greater than that of the surface. As a result, the diffuse layer will see a high concentration of co-ions, instead of counterions, close to the surface. Overcharging explains, among other effects, why proteins adsorb to a charged surface of the same polarity (same sign). The observations can be justified based on chemico-physical considerations, but cannot be explained by the classical mean-field models ${ }^{101,129-143}$, because they do not account for co-ions.

Sum-frequency generation (SFG, see BOX 5) spectroscopy measurements at the aqueous interface of a model membrane featuring different lipids with opposite charges showed that counterions move close to the membrane surface to screen the charge and form a Stern layer ${ }^{144}$, as also commonly observed for hard surfaces ${ }^{145}$. However, in the case of phospholipid membrane, when the charge density increases even more, counterions can penetrate into the headgroup region, not only neutralizing the lipid charge but also causing the rearrangement of lipids and interfacial water (FIG. 4). The counterion 
charge compensation, ensuring that the total surface charge does not exceed a specific critical value ${ }^{104}$, can be well described by classical mean-field models. This has also been reported for liposomes when mixing zwitterionic and negatively charged phospholipids ${ }^{146,147}$. On the contrary, the counterion penetration in the charged lipid monolayer cannot be described using classical models ${ }^{148,149}$ but requires a molecular description, such as that provided by molecular dynamics (MD) simulations ${ }^{144}$. Incidentally, charge saturation can explain why nature limits the amount of negatively charged phospholipids in membranes.

Mean-field models, even with the refinements discussed earlier, consider the ions as point charges interacting through electrostatics. This leads to underestimating or overestimating screening and the inability to predict phenomena such as ion-specific effects, overcharging and charge inversion, the matching water affinity law and surface restructuring that have experimentally been well documented.

\section{Water structuring and orientation}

Water has a strong dipole moment. As a result, the presence of charge on a surface will lead to an average preferential orientation of water molecules near the interface. This preferential alignment of water molecules is dynamically and spatially heterogeneous.
Most experimental techniques average over molecular length scales and timescales, and typically provide access to ensemble-averaged water alignment. It is important to note that, in addition to a dipole, water molecules have the propensity to form $\mathrm{H}$-bonds that are responsible for its many peculiar properties ${ }^{150-152}$. Water's ability to form $\mathrm{H}$-bonds explains its strong interaction with oxides and biological systems. On metal surfaces, because the water adsorption energy is comparable to the $\mathrm{H}$-bond strength, the competition between water-water and water-surface interactions can give rise to very different interfacial water structures, depending on the specific system under consideration ${ }^{30,32,153}$.

The first experimental evidence for oriented interfacial water at a positive ( $O$ pointing towards the surface) versus a negative ( $\mathrm{H}$ pointing towards the surface) surface was reported for charged surfactants forming monolayers at the air-water interface using SFG ${ }^{154}$ (BOX 5). Soon after, it was also reported for phospholipids ${ }^{155,156}$ and confirmed in several studies ${ }^{120,144,157-159}$. Changes of the interface charge density through fine-tuning of the solution's $\mathrm{pH}$ was also found to affect the orientation extent of the water molecules, as has been observed on silica surfaces ${ }^{80,116,160,161}$. In this context, water orientational asymmetry is an example in which the shortcomings of the classical mean-field models become immediately apparent. A positive charge typically aligns

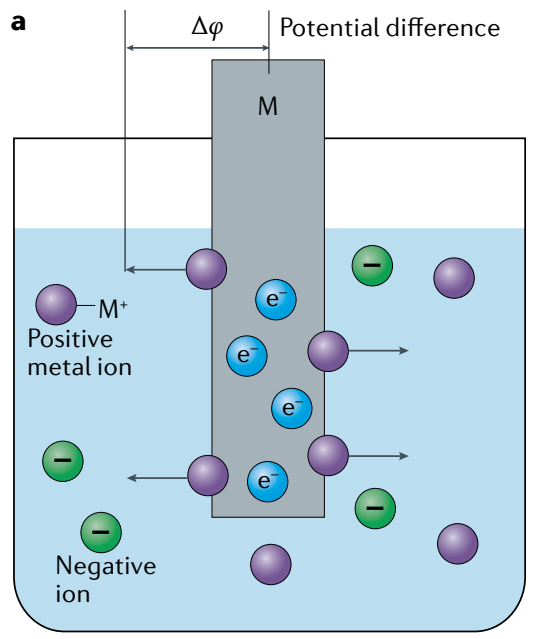

b

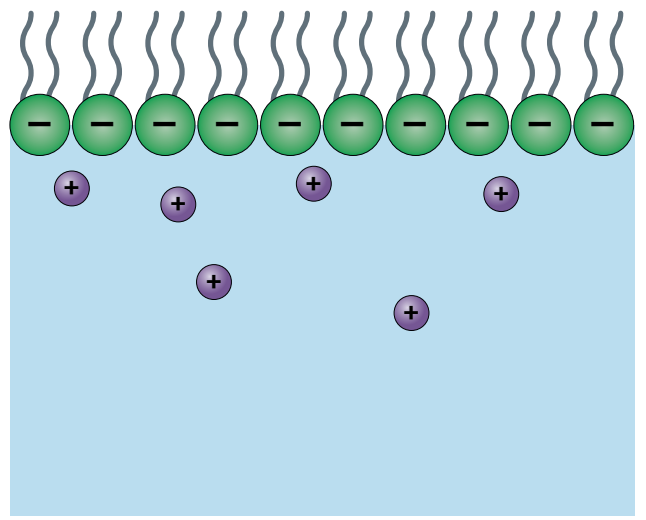

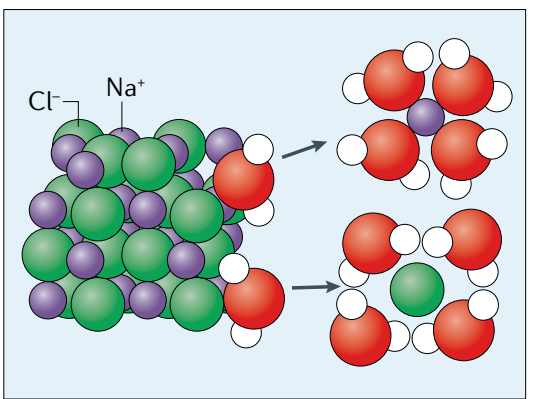

d

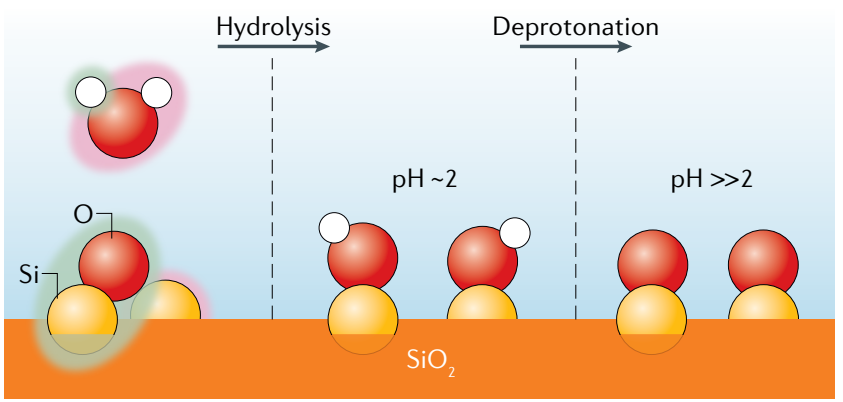

Fig. 3 | Possible sources of surface charge. Charge generated by an applied voltage to an electrode (part a), counterion dissolution and/or counterion physisorption or protonation/deprotonation at a biomembrane (part b), ion dissolution from an ionic crystal (such as $\mathrm{NaCl}$ ) (part c) and water hydrolysis at a siloxane bridge followed by deprotonation at increased $\mathrm{pH}$ (part d). 


\section{Box 4 | Photoelectron spectroscopy}

Photoelectron (photoemission) spectroscopy (PES) is based on two main electron emission processes: that of direct photoelectrons (see the figure, part a, left) and that of electrons created in some second-order process, including Auger decay and several non-local autoionization processes, such as intermolecular Coulombic decay (see the figure, part a, right) ${ }^{281}$. Direct photoionization gives access to electron binding energies of the water solvent, the solutes in bulk solution, as well as those at the solidaqueous solution interface.

This process also informs us on valence energies ${ }^{282}$, important a

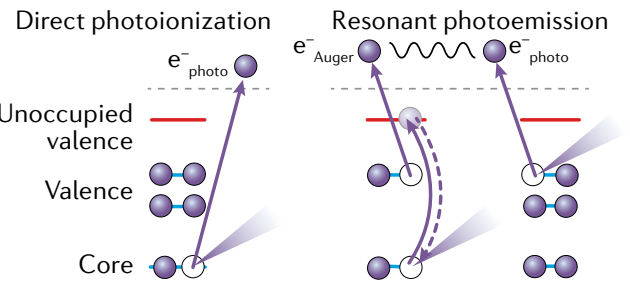

b

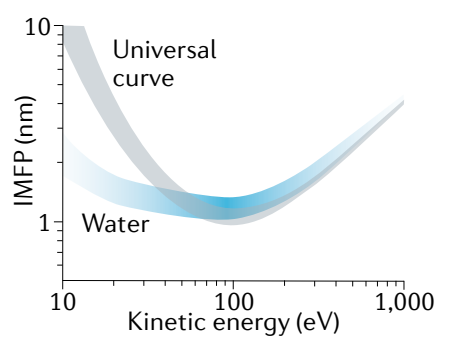

for chemical reactivity in aqueous solution, and core-level energies (and associated chemical shifts), which report on local structure and charge ${ }^{262}$. The second-order processes provide insight into water molecular structure and dynamics thanks to their relationship to the dynamical local hydrogen-bonding networks. Moreover, for resonant PES, Auger decay leads to the same final state reached in direct ionization, and interference of the identical final states results in a resonant enhancement ${ }^{260,261}$. This can be exploited to increase the detection sensitivity of low-concentration species undetectable in the off-resonant PES spectra. Resonant PES allows measuring X-ray absorption, based on total electron yields obtained by integrating the PE spectra over a suitable electron kinetic energy range ${ }^{283}$.

PES is a surface-sensitive technique, but accessing buried interfaces remains challenging ${ }^{284}$. Buried interfaces can be accessed in the case of nanoparticles dispersed in aqueous solution using a microjet and solid samples covered with few monolayers of water, stabilized at high relative humidity. The probing depth depends on the kinetic energy of the photo and Auger electron: slower bulk electrons scatter more before escaping into vacuum than higher kinetic energy ones. Maximum surface sensitivity is reached at a kinetic energy of $\sim 100 \mathrm{eV}$, at which the electron inelastic mean free path (IMFP) is shortest. For lower energies, the IMFP usually increases, although for liquid water, such an increase seems to be much smaller (see the figure, part b). This explains the challenge of measuring PES from solid substrates in contact with bulk liquid water.

Part $\mathbf{a}$ adapted with permission from REF. ${ }^{261}$, American Chemical Society. Part $\mathbf{b}$ adapted with permission from REF ${ }^{280}$, American Physical Society.

the water dipole, lined up with the bisector of the two $\mathrm{OH}$ bonds within the water molecule, as has previously been shown for water hydrating ions ${ }^{162}$. Yet, a negative charge can also act as an $\mathrm{H}$-bond acceptor, aligning only one of the $\mathrm{OH}$ bonds, rather than the dipole of the water molecules ${ }^{163,164}$.

For interfaces composed of differently charged lipids described in the previous section (FIG. 4), the degree of water alignment scales with the surface charge - but only for very low lipid charge densities (FIG. 4a,b). When mixing positively and negatively charged lipids while keeping their total number constant, it is observed that, with further increase of the surface charge beyond a certain threshold charge density, the water alignment saturates (dielectric saturation), meaning that, once the near-surface water molecules are aligned, no further changes in water alignment can be induced, even if the field is further increased ${ }^{144,146}$. It is also worth pointing out that the water-lipid interface is much less sharp than, for instance, the water-air or water-solid interfaces.
Therefore, in the case of water-lipid interfaces, the density of water molecules decreases less abruptly when moving from the bulk towards the lipid alkyl chains. Water has been found to form $\mathrm{H}$-bonds not only with headgroups but also with carbonyl groups that are located between the hydrophobic alkyl chains and the hydrophilic headgroup region ${ }^{165-167}$. However, substantial water orientation has also been observed for zwitterionic, overall neutral lipids. The net average orientation of interfacial water changes by $\sim 180^{\circ}$ following the chemical inversion of phosphate $(\mathrm{P})$ and choline $(\mathrm{C})$ in the headgroup (for example, from $\mathrm{P}-\mathrm{C}$ to $\mathrm{C}-\mathrm{P}$ ). Here, the force orienting the water molecules results from the local dipolar field generated between the positive $\mathrm{C}$ and negative $\mathrm{P}$ groups ${ }^{156,168-170}$ and, presumably, H-bonding interactions in the headgroup region ${ }^{67,171}$.

Also, for conducting electrodes, experimental measurements ${ }^{172-175}$, as well as atomistic scale modelling $^{33,174,176,177}$, have confirmed that the orientation of water is field-dependent and potential-dependent (FIG. 5a). The $\mathrm{H}$ atoms of the water molecule point towards the surface at low potentials $\left(E<E_{\mathrm{pzc}}\right)$ and $\mathrm{O}$ points towards the surface at potentials above the potential of zero charge $\left(E>E_{\mathrm{pzc}}\right)^{178}$. Further, the density of water near the surface is much higher than that of the bulk at all potentials, which implies a different ordering of water molecules at the interface ${ }^{179}$. Comparison between electron-yield X-ray absorption spectra (BOX 4) of interface and bulk liquid water reveals distinct types of interfacial water molecules in contact with a polycrystalline gold surface, different from those in the bulk electrolyte. Specifically, undercoordinated (single H-bond donating) water molecules exist at the surface, with their non-H-bonded $\mathrm{OH}$ group suggested as being either parallel or perpendicular to the surface ${ }^{174,175}$.

Studies at charged lipid or surfactant monolayers reveal that not only the water structure but also the vibrational relaxation dynamics of interfacial water is affected by the presence of the interface ${ }^{180}$. In particular, the dynamics seem to depend on the sign of the surface charge. For positive monolayers, the dynamics of interfacial water closely resembles that of bulk water and that at the air-water interface. Even though for negative monolayers an agreement on the interpretation of the data has not yet been reached, all available results suggest the dynamics to be different from that of the positive interfaces, probably because of the strong $\mathrm{H}$-bond formed with the negative group in the polar head ${ }^{181-183}$. This observation underlines the aforementioned asymmetry of water in response to positively and negatively charged surfaces. Interestingly, water dynamics in contact with zwitterionic lipids do not seem to be affected by the inversion of phosphate and choline ( $\mathrm{P}-\mathrm{C}$ versus $\mathrm{C}-\mathrm{P}$ ) in the headgroup ${ }^{184}$. The effect of the surface charge has also been observed in the case of silica, where the vibrational lifetime of $\mathrm{H}$-bonded $\mathrm{OH}$ dramatically shortens when the surface charge increases (high $\mathrm{pH})^{185}$. The reorientational dynamics of interfacial water has been studied at extended neutral surfaces ${ }^{186,187}$, but not yet for charged interfaces. Such experiments would be very informative for better understanding interfacial dynamics and local dielectric constants. 
Besides charge-induced alignment of water directly at the interface, the interaction of the first molecular water layer at the interface can have consequences for the structure of water farther away. X-ray reflectivity experiments revealed oscillations in the water $\mathrm{O}$ density, in the direction normal to the surface away from a mica-water interface ${ }^{188}$ (FIG. 6a,b). Despite the soft nature of biomembranes, scanning force microscopy (BOX 3) has been successfully applied to such interfaces, demonstrating layering of water ${ }^{189}$ (FIG. 6c), which has also been observed for minerals ${ }^{190}$. Local imaging with scanning probe microscopy shows both vertical layering of water and a lateral structure of the calcite $\left(\mathrm{CaCO}_{3}\right)$-water interface ${ }^{191}$, known as a 'checkerboard' pattern (FIG. $\left.6 \mathrm{~d}, \mathrm{e}\right)$. The checkerboard pattern agrees well with predictions from MD simulations finding that the water closest to the surface is bound through its electronegative $\mathrm{O}$ atom to the surface calcium cation, while the water in the next layer resides above a carbonate site, forming an $\mathrm{H}$-bond to the protruding $\mathrm{O}$ atom of the surface carbonate group $^{192}$. This arrangement is associated with a switch in the water orientation in the first and second layers, respectively. From the atomic force microscopy (BOX 3)

\section{Box $5 \mid$ Surface-sensitive nonlinear optical techniques}

Nonlinear optics typically involves the frequency conversion of optical fields by a nonlinear interaction with a material. In vibrational sum-frequency generation (SFG) spectroscopy, an infrared (IR) and a visible (VIS) pulsed laser beam are overlapped in space and time at an interface, generating photons at the sum-frequency of the two incident frequencies. Vibrational information can be obtained by tuning the IR frequency with a vibrational mode ${ }^{285-287}$. Second-harmonic generation (SHG) is a degenerate case of SFG in which only one laser beam is used and photons at twice the frequency of the incident laser beam are generated. As with any spectroscopy, SFG and SHG have selection rules. A key selection rule for SFG and SHG is that symmetry must be broken, which happens at the interface between two media. Water at a charged interface has been extensively studied using both techniques. In SFG, the IR frequency is usually tuned to be resonant with the $\mathrm{OH}$ stretching mode ${ }^{29}$. A schematic representation of SFG spectroscopy at a charged phospholipid monolayer-water interface in reflection geometry can be seen in part a of the figure. In parts $b$ and $\mathbf{c}$ of the figure, the level diagrams for SFG and non-resonant SHG are shown. Additionally, in SFG, the frequency of the $\mathrm{OH}$ signal reports on the hydrogen bonding strength, while the intensity reports on the molecular ordering. A charged interface generates a field into the solution. Its depth, at low ionic strength, equals the Debye length (FIG. 1) and depends on the ionic strength and species. This static field induces a reorientation of water molecules, so as to minimize their dipole energy, and, possibly, a change in their polarizability. Both phenomena effectively break the symmetry in the near-interface region, leading to enhanced SFG or SHG signals ${ }^{88,90,156,163,169,276,288,289}$. With the use of appropriate models, the signals can be converted into surface potential ${ }^{80,82,84,157,159,254,267-275,290,291}$. NIR, near infrared.
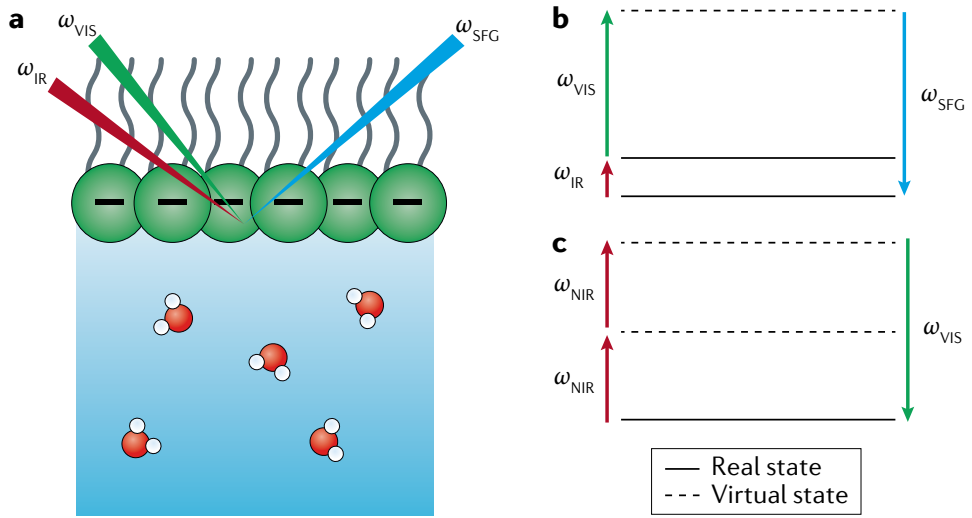

data, the water dipole orientation cannot be deduced, but SFG can provide this information. If the MD prediction of oppositely oriented water molecules is correct, this should result in local restoration of centrosymmetry and, thus, a very small SFG signal from the interfacial water. The layering behaviour at interfaces described above for water can also be observed for hydrated ions ${ }^{193}$.

Water orientational asymmetry and layering cannot be described by classical mean-field models. Therefore, it is apparent from the discussion in this section that, to reach a deeper understanding of the phenomena happening at the interface, water cannot be simply considered as a continuous homogeneous medium defined by its permittivity or even as a simple dipole. Rather, its charge-asymmetrical molecular structure at the interface and the ability to accept and donate hydrogen bonds need to be accounted for in many cases.

\section{Modern electrical double layer theories}

To this point, we have addressed the classical mean-field theories of charged aqueous interfaces, discussed each component of the system and pointed out several observations that suggest that this classical description is qualitatively incomplete. Along with the development of in situ experimental techniques that allow looking at the water-ion-charged interface in more (sometimes even atomic) detail ${ }^{55,91,144,146,154,164,189,194,195}$, simulations have allowed the study of the breakdown of the classical mean-field theories at the molecular length scale. This has led to the successful development of modified mean-field approaches. For example, correlation effects are not captured by mean-field theories, but simulations have shown that they become important when the electrostatic coupling parameter, $\Xi=2 \pi \sigma l_{\mathrm{B}}^{2} q^{3} / e$, exceeds 10 (where $\sigma, q$ and $e$ are the surface charge density, the ion valence and the electron charge, respectively $)^{142}$. The Bjerrum length, $l_{\mathrm{B}}=e^{2} /\left(4 \pi \varepsilon_{0} \varepsilon k_{\mathrm{B}} T\right)$, is the distance at which the Coulomb interaction energy between two monovalent ions equals the thermal energy $k_{\mathrm{B}} T$. At physiological electrolyte concentrations, the Debye and Bjerrum lengths are similar and equal to $\sim 1 \mathrm{~nm}$. For monovalent ions, the threshold $\Xi=10$ is reached for $\sigma=1.6 \mathrm{e} \mathrm{nm}^{-2}$, which is a very high surface charge density. For divalent ions, correlation effects become important already at $\sigma=0.2 \mathrm{enm}^{-2}$, which is a rather modest value readily reached in many systems. What happens when the mean-field model breaks down? The mean-field theory predicts repulsion between similarly (same sign) charged surfaces, regardless of their surface charge density. In reality, in the strong coupling regime $(\Xi>>10)$, similarly charged surfaces attract each other ${ }^{142}$. This explains why DNA and highly charged minerals condense in the presence of multivalent ions ${ }^{133}$.

Even for moderately charged surfaces, additional corrections to mean-field theory owing to the discrete water nature and specific ion-surface interactions can be important. To illustrate this, we compare predictions of mean-field theory with MD simulations of a charged surface that include explicit water molecules. We note in passing that MD simulations have their own limitations, in particular, with regards to the employed force fields, which will be discussed in the 'Outlook' section. 

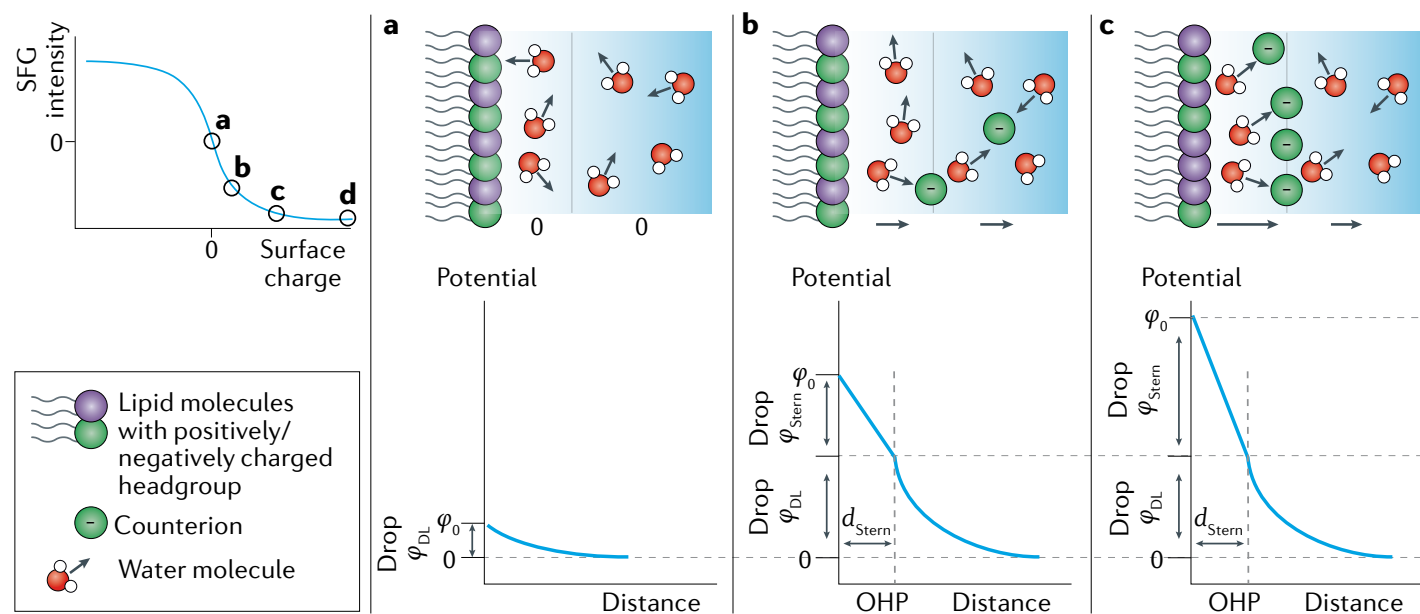

Potential
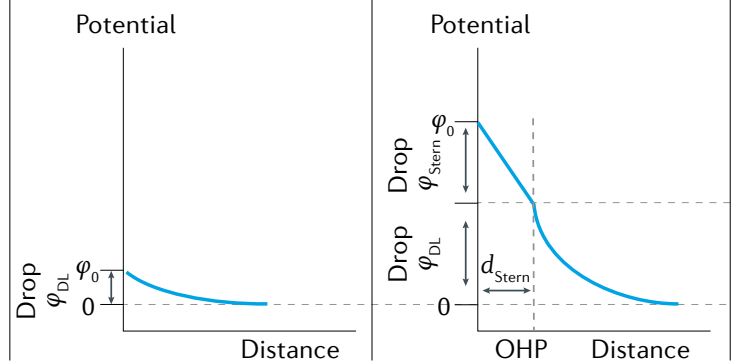

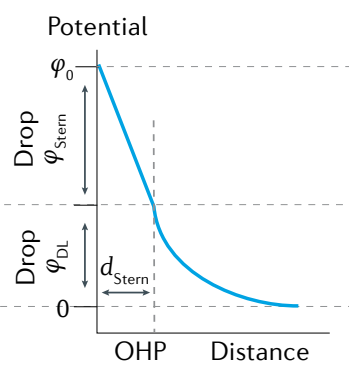

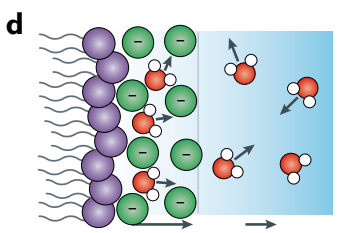

Potential

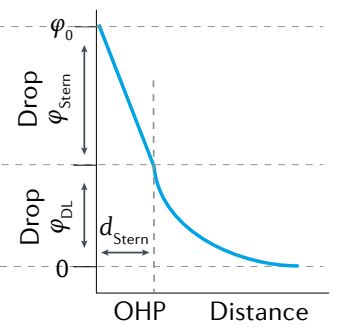

Fig. 4 | Water at charged biomembranes. Water orientation, counterion distribution and surface potential as a function of the net charge of a mixed phospholipid monolayer featuring positively charged 1,2-dipalmitoyl-3-trimethylammoniumpropane (DPTAP) and negatively charged 1,2-dipalmitoyl-sn-glycero-3-phospho-(1'-rac-glycerol) sodium salt (DPPG) ${ }^{144}$. The initial behaviour as a function of surface charge can be described by mean-field models (parts $\mathbf{a}$ and $\mathbf{b}$ ). At higher charge $\left(\sim 0.1 \mid \mathrm{C} \mathrm{m}^{-2}\right)$, the surface potential saturates because of counterion adsorption (part c), followed by lipid rearrangement at even higher charge densities (part d), both not captured by classical mean-field models. $\varphi_{0}$, surface potential; Drop $\varphi_{\mathrm{DL}}$, potential drop across the diffuse layer; $\operatorname{Drop} \varphi_{\text {Stern }}$, potential drop across the Stern layer; $d_{\text {Stern }}$, thickness of the Stern layer; OHP, outer Helmholtz plane; SFG, sum-frequency generation. Reprinted with permission from REF. ${ }^{144}$, AAAS.

FIGURE 7a shows a simulation snapshot of two negatively charged decanol surfaces, while the counterion density profile for a surface charge density $\sigma=-0.11 \mathrm{enm}^{-2}$ and surface separation $d=6 \mathrm{~nm}$ is given in FIG. $7 \mathrm{~b}\left(\mathrm{REF}^{178}\right)$. The standard mean-field prediction, which assumes surface charges that are sharply localized on two plates and only electrostatic ion-surface interactions, deviates substantially from the MD results. The modified mean-field theory includes an additional non-electrostatic ion-surface interaction potential $U(z)$, which brings the simulated profile in close agreement with the MD profile $\mathrm{e}^{178}$. The ion-surface interaction $U(z)\left(\mathrm{REF}^{178}{ }^{178}\right.$ (FIG. $7 \mathrm{C}$ ) is repulsive and decays exponentially over $1 \mathrm{~nm}$. It accounts for direct, ion-specific ion-surface interactions and for the finite ion size, all effects that are not accounted for in the classical mean-field models. The orange line in FIG. $7 \mathrm{C}$ shows the surface charge distribution, which is rather delocalized and can also be included in the modified mean-field model ${ }^{178}$. We conclude that microscopic insight from simulations allows designing a much-improved model based on mean-field theories.

FIGURE $7 \mathrm{~d}$ shows the water orientation profiles for different surface charge densities, together with the water density profile ${ }^{178}$. For zero surface charge, interfacial water molecules point their oxygen atoms to the surface to form hydrogen bonds with the surface hydrogens. Surprisingly, already at a low surface charge density of $\sigma=-0.11 \mathrm{enm}^{-2}$, the water molecules near the surface reorient over a rather long spatial range $(\sim \mathrm{nm})$ equivalent to the Debye length. It is known that the water dielectric constant becomes tensorial at surfaces with pronounced differences in the direction parallel and perpendicular to the surface ${ }^{196}$. The results in FIG. $7 \mathrm{~d}$ demonstrate that surface charges reshape the hydration water structure, calling once more into question the use of the bulk dielectric constant of water at charged surfaces.
The intricate coupling between interfacial water structure, surface charge and dielectric effects can be illustrated by the simulation of an even simpler system, namely, water between two flat graphene layers, a snapshot of which is shown in FIG. 7e (REFS ${ }^{197,198}$ ). The interfacial water density profile in FIG. $7 \mathrm{f}$ shows the well-known and expected pronounced water layering at such a rigid surface. FIGURE $7 g$ presents the intrinsic electric field profile $E_{0}(z)$ for a neutral graphene sheet, which is solely produced by the water polarization, and the electric field profile $E(z)$ in the presence of an external displacement field of strength $D / \varepsilon_{0}=1 \mathrm{~V} \mathrm{~nm}^{-1}$, which corresponds to the field produced by a moderate surface charge of about $\sigma=+0.1 \mathrm{enm}^{-2}$. The $E_{0}(z)$ profile reaches high values of $10 \mathrm{~V} \mathrm{~nm}^{-1}$ in the first hydration layer, but $E_{0}(z)$ and $E(z)$ are quite close to each other. From the difference between these two fields, we can calculate the inverse dielectric profile according to $1 / \varepsilon(z)=\varepsilon_{0}\left(E(z)-E_{0}(z)\right) / D$ (REF. ${ }^{198}$ ) (FIG. $\left.7 \mathrm{~g}\right)$. Notably, $1 / \varepsilon(z)$ changes its sign (indicating divergences of $\varepsilon(z)$ ) and does not seem to be related to the intrinsic electric field $E_{0}(z)$ or to the water density profile in FIG. $7 \mathrm{f}$. The dielectric profile $1 / \varepsilon(z)$ derived within the linear response theory agrees very nicely with the finite-field result ${ }^{198}$ (FIG. $7 \mathrm{~g}$ ), which demonstrates that linear response is valid for surface charge densities on the order $\sigma=0.1 \mathrm{enm}^{-2}$. This is interesting because the water

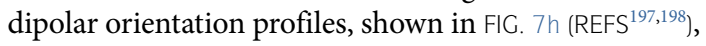
indicate pronounced layered orientation and are quite different for neutral and charged surfaces. This demonstrates the robustness of the dielectric linear response, which is embedded in the dielectric profile, the effects of which can also be included in modified mean-field models ${ }^{178}$. For the larger surface charge densities shown in FIG. $7 d$, the dielectric water profile becomes modified and nonlinear dielectric effects must eventually be taken into account. 

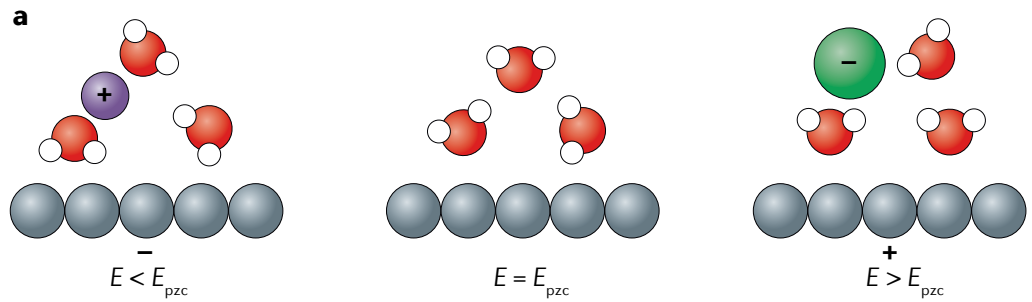

b

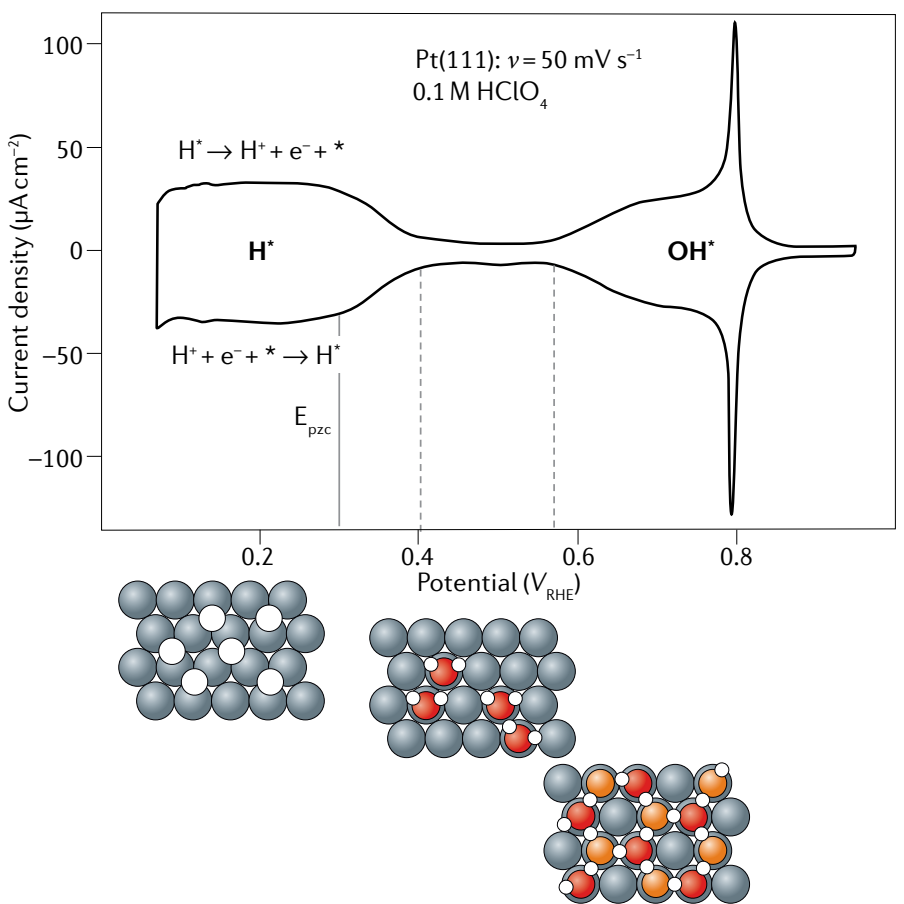

C

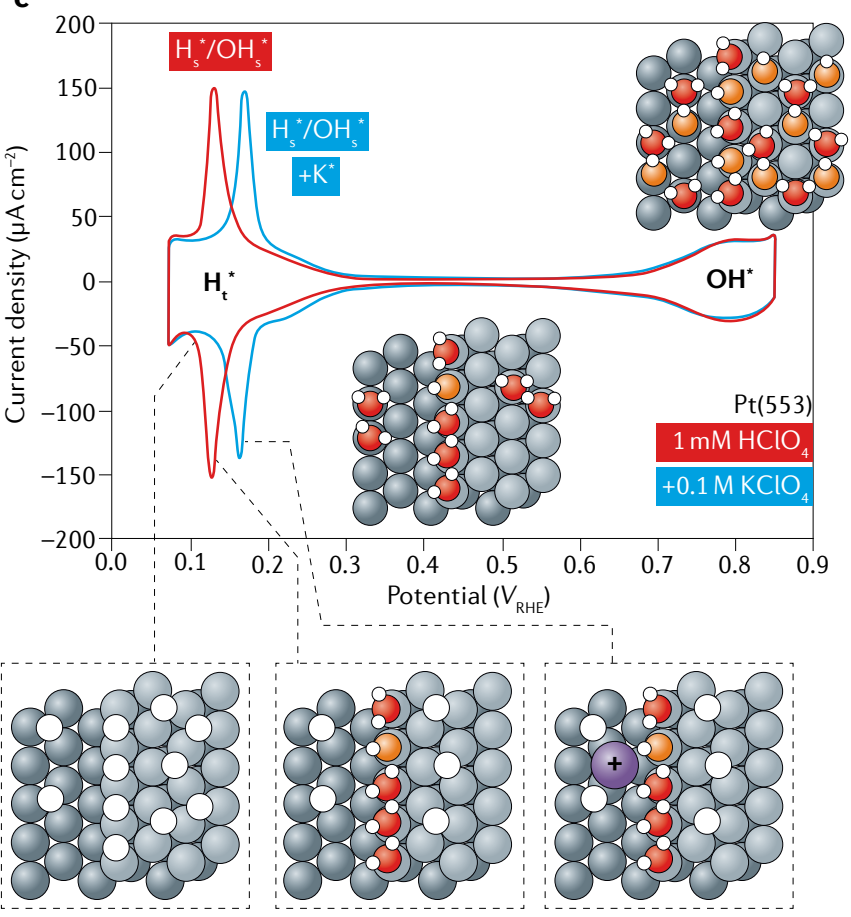

Fig. 5 | Water at a charged electrode interface. a | Variation of the structure of water and ion concentration at the electrode-electrolyte interface with potential $(E)$ relative to the potential of zero charge $\left(E_{\mathrm{pzc}}\right) \cdot \mathbf{b} \mid$ Cyclic voltammogram measured on $\mathrm{Pt}(111)$ in $0.1 \mathrm{M} \mathrm{HClO}_{4}$, illustrating the potential regions where hydrogen $\left(\mathrm{H}^{\star}\right)$, water $\left(\mathrm{H}_{2} \mathrm{O}^{*}\right)$ and hydroxide $\left(\mathrm{OH}^{\star}\right)$ adsorb onto the electrode surface ${ }^{237}$. c | Cyclic voltammograms measured on $\mathrm{Pt}(553)$ in $1 \mathrm{mM} \mathrm{HClO}_{4}$ (red) and $1 \mathrm{mM}$ $\mathrm{HClO}_{4}$ with $0.1 \mathrm{M} \mathrm{KClO}_{4}$ (blue) ${ }^{228}$. Although hydrogen and hydroxide adsorption on the (111)-like terrace sites occurs at similar potentials to on the low-index $\mathrm{Pt}(111)$ surface, adsorption of these species at the undercoordinated step $\left(\mathrm{H}_{\mathrm{s}}{ }^{*}\right.$ and $\mathrm{OH}_{\mathrm{s}}{ }^{*}$ ) sites is much stronger, such that the region where hydrogen and hydroxide adsorb overlap at the step edge. Further, the adsorption of an alkali metal cation at the step edge acts to weaken hydroxide adsorption (by disrupting strong hydrogen bonds between $\mathrm{OH}^{\star}$ and $\mathrm{H}_{2} \mathrm{O}^{\star}$ ), shifting the sharp, step-associated peak to more positive potentials. For both electrodes, density functional theory has been used to determine at what potentials each species adsorbs as a function of concentration in solution, potential and coverage on the surface ${ }^{227,228,231}$. RHE, reversible hydrogen electrode. Part $\mathbf{b}$ adapted with permission from REF. ${ }^{237}$, Royal Society of Chemistry. Part $\mathbf{c}$ adapted with permission from REF. ${ }^{228}$, Wiley.

In recent years, force-field-based simulation techniques have been developed to include effects of constant potential and constant-field boundary conditions ${ }^{179,199-201}$, which are important at metallic surfaces. Likewise, ab initio simulation techniques can, nowadays, treat charged surfaces, including chemical reactions with surface water molecules ${ }^{202,203}$, but are limited to relatively small systems and can, therefore, not yet account for the diffuse ionic double layer. We conclude that the hydration water structure at charged surfaces exhibits pronounced water orientations that lead to a strong intrinsic electric field at interfaces and is characterized by an oscillatory dielectric response profile. Surface charges affect the water orientation profile (as seen in FIG. 7d) and, thereby, modify water polarization and dielectric response. This clearly demonstrates the complexity of hydration water at charged surfaces and the need to account for water's molecular properties theoretically, for example, through the combined use of simulations and modified mean-field approaches ${ }^{178,204-208}$.

\section{Interfacial reactivity}

When an aqueous electrolyte solution meets a charged surface, the EDL formation (FIG. 1) is not the only result: chemical reactions usually occur. While reactions are the interesting product of this encounter, this 'chemistry' eludes both classical mean-field models and force-field-based classical MD simulations.

The breakdown of classical mean-field models is particularly evident when considering minerals for which water does not only adsorb on their surface molecularly but can also undergo (electron-induced or 
photo-induced) reactions. For example, water adsorbs dissociatively (into $\mathrm{H}^{\star}$ and $\mathrm{OH}^{\star}$ groups) and subsequently reacts to form $\mathrm{H}_{2}$ and $\mathrm{O}_{2}$. As shown in FIG. 5b, at an electrochemical interface, water can decompose to form adsorbed hydrogen, hydroxide and oxygen, with a transfer of electrons to or from the electrode surface. The adsorption of these water decomposition products $\left(\mathrm{H}^{*}, \mathrm{OH}^{*}\right.$ and $\left.\mathrm{O}^{*}\right)$ depends on the electrode potential, composition and surface structure ${ }^{209-212}$.

In photocatalysis, the $\mathrm{TiO}_{2}$-water interface has remained at the forefront of research for many years, owing to its seminal role in water splitting ${ }^{16,17}$. Whereas water from the vapour phase interacting with $\mathrm{TiO}_{2}$ has been investigated extensively using various approaches in ultra-high vacuum ${ }^{213,214}$, studies of the interaction of $\mathrm{TiO}_{2}$ with bulk water have been lagging ${ }^{215}$. This can be traced to the challenge of studying the buried oxide-water interface using traditional surface science approaches. For the (110) rutile facet, a pioneering work pointed out the importance of combining molecular scale (ab initio calculations and MD simulations) and macroscopic approaches (GCS model). This combined approach aimed to give a comprehensive picture of the interface probed with a suite of experimental techniques, to predict ion distributions not accessible experimentally and to establish the presence of a single interfacial water layer with molecules both laterally and vertically ordered with respect to the rutile structure ${ }^{216}$. In a step intermediate between $\mathrm{TiO}_{2}$ in ultra-high vacuum and in contact with bulk water, photoelectron spectroscopy was performed on (mixed rutile and anatase) $\mathrm{TiO}_{2}$ nanoparticles exposed to water vapour ${ }^{217}$. These studies revealed the possibility of dissociative adsorption of water on the O-terminated surface ${ }^{218}$ to result in the formation of two adjacent (paired) hydroxyl groups ${ }^{219,220}$, with a very small energy difference between adsorption of intact $\mathrm{H}_{2} \mathrm{O}$ and dissociative adsorption.

Using resonant photoemission spectroscopy, molecularly adsorbed $\mathrm{H}_{2} \mathrm{O}$ molecules have been observed on the surface of $\mathrm{TiO}_{2}$ nanoparticles in acidic solution, whereas a dissociative water interaction, leading to $\mathrm{OH}$ species at the $\mathrm{TiO}_{2}$ surface, was observed for near-neutral solutions ${ }^{60}$. These results suggest that, at acidic $\mathrm{pH}$, the protonation of adsorbed $\mathrm{OH}$ at the Ti site of the defect-free surface of anatase nanoparticles is fast, leading to molecularly adsorbed water being the dominant species. By contrast, at basic $\mathrm{pH}$, the protonation of the surface $\mathrm{OH}$ group is suppressed, which makes the

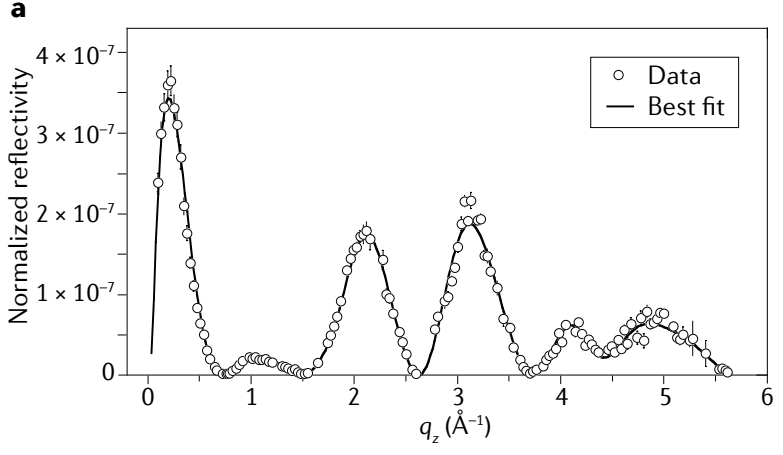

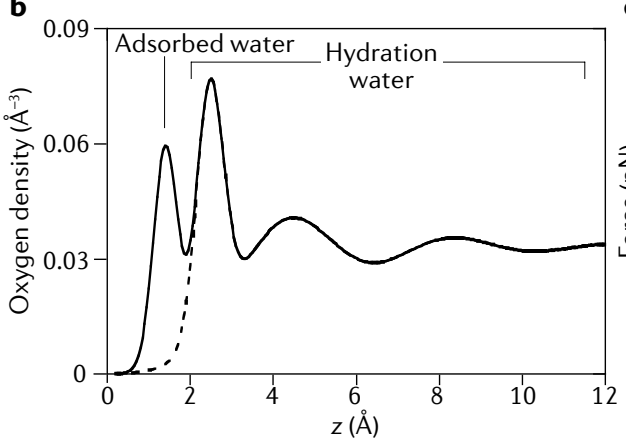

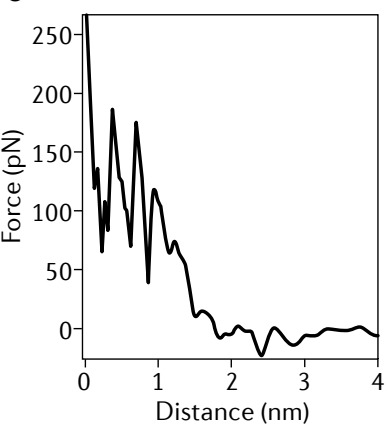

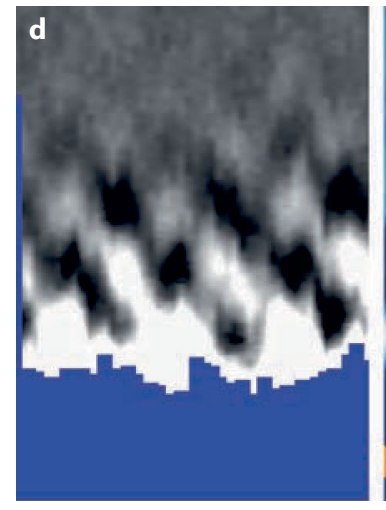

Fig. 6 | Layering of water at biomembranes and mineral surfaces. a | Normalized X-ray reflectivity from the mica-water interface. $\mathbf{b}$ |Density distribution of the interfacial water oxygen atoms as a function of the distance from the surface as obtained from the fit of the data in part $\mathbf{a}$. The large $z$-limit value of 0.033 atoms $\AA^{-3}$ corresponds to the mean oxygen density of bulk water. c|Oscillatory hydration force measured as a function of the distance from the gel-phase 1,2-dipalmitoyl-sn-glycero3-phosphocholine (DPPC) bilayer surface: each oscillation corresponds to the displacement of a water layer. Thus, the periodicity of the oscillations relates to the size of the water molecule. $\mathbf{d}$ | Lateral slice $(x z)$ through a $3 \mathrm{D}$ atomic force microscopy map collected at the calcite-water interface ${ }^{241}$.

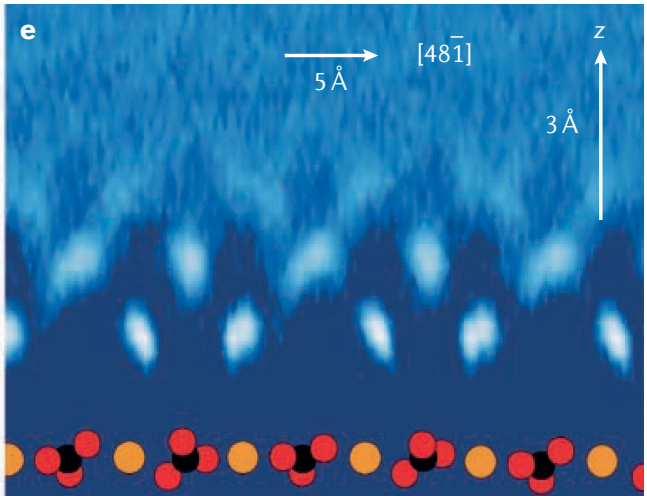

In this slice, the excitation frequency shift is shown. Using the solvent-tip approximation ${ }^{242,243}$, bright features can be associated with high water density. e | Molecular dynamics simulations for the same calcite-water system illustrating the water arrangement at the interface ${ }^{244}$. The density of the water oxygen atoms is shown. Both atomic force microscopy and molecular dynamics results show the characteristic checkerboard pattern of the hydration structure at the calcite surface. Parts $\mathbf{a}$ and $\mathbf{b}$ reprinted with permission from REF. ${ }^{188}$, American Physical Society. Part c reprinted with permission from REF. ${ }^{189}$, Elsevier. Part $\mathbf{d}$ adapted with permission from REF. ${ }^{245}$, American Institute of Physics. Part e reprinted with permission from REF. ${ }^{244}$, American Chemical Society. 


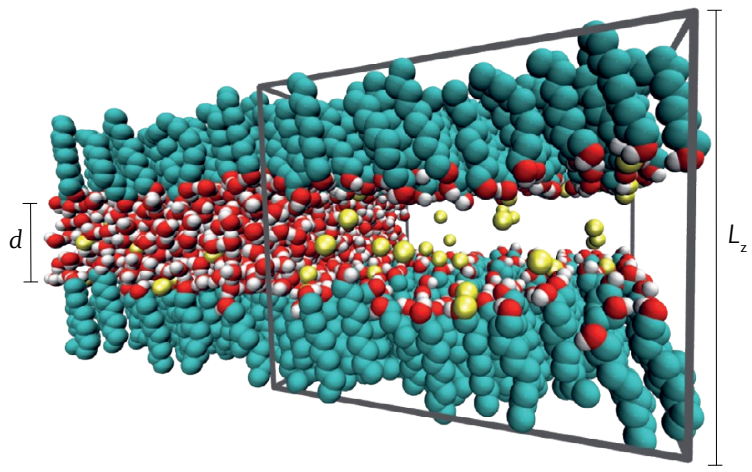

b

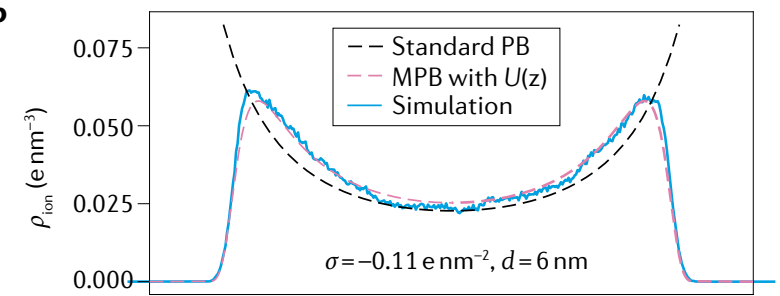

c

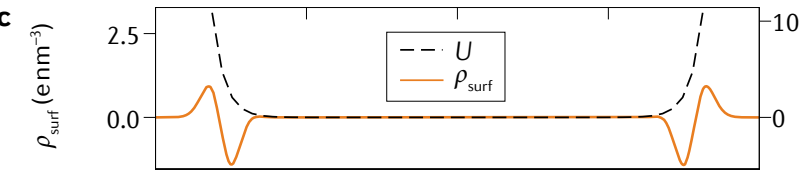

d

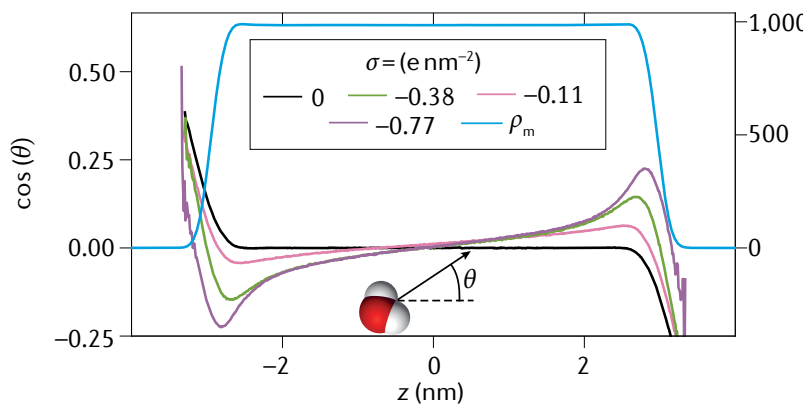

Fig. 7 Molecular dynamics simulations of water at charged interfaces. a | Molecular dynamics simulation snapshot of two interacting, negatively charged decanol self-assembled monolayer (SAM) surfaces at a separation $d$ including explicit water. The neutralizing $\mathrm{Na}^{+}$ions are represented by yellow spheres. Water is not shown in the centre of the simulation box for clarity. $\mathbf{b} \mid$ Comparison of the simulated counterion density profile $\left(\rho_{\text {ion }}\right)$ (blue line) with the standard mean-field (black line) and a modified mean-field model (pink line) that includes the ion-SAM interaction potential U(z). All calculations correspond to a surface charge $(\sigma)$ of $-0.11 \mathrm{enm}^{-2}$ and a surface separation distance, $d$, of $6 \mathrm{~nm}$ (panel a); all profiles are symmetrical around the middle of the water slab at $z=0$. The potential $U(z)$ is seen to be crucial for an accurate description of the simulated density profile. $c \mid$ The surface charge density profile $\left(\rho_{\text {surf }}\right)$ (orange line) is significantly smeared out, which is neglected in standard mean-field models. The ion-SAM interaction potential $U(z)$ (black line) is repulsive and decays exponentially moving away from the SAM surface. $\mathbf{d}$ / Water orientation profiles for different surface charge densities, indicating water reorientation already for modest surface charges. The blue line denotes the water mass density profile $\left(\rho_{\mathrm{m}}\right)$, e
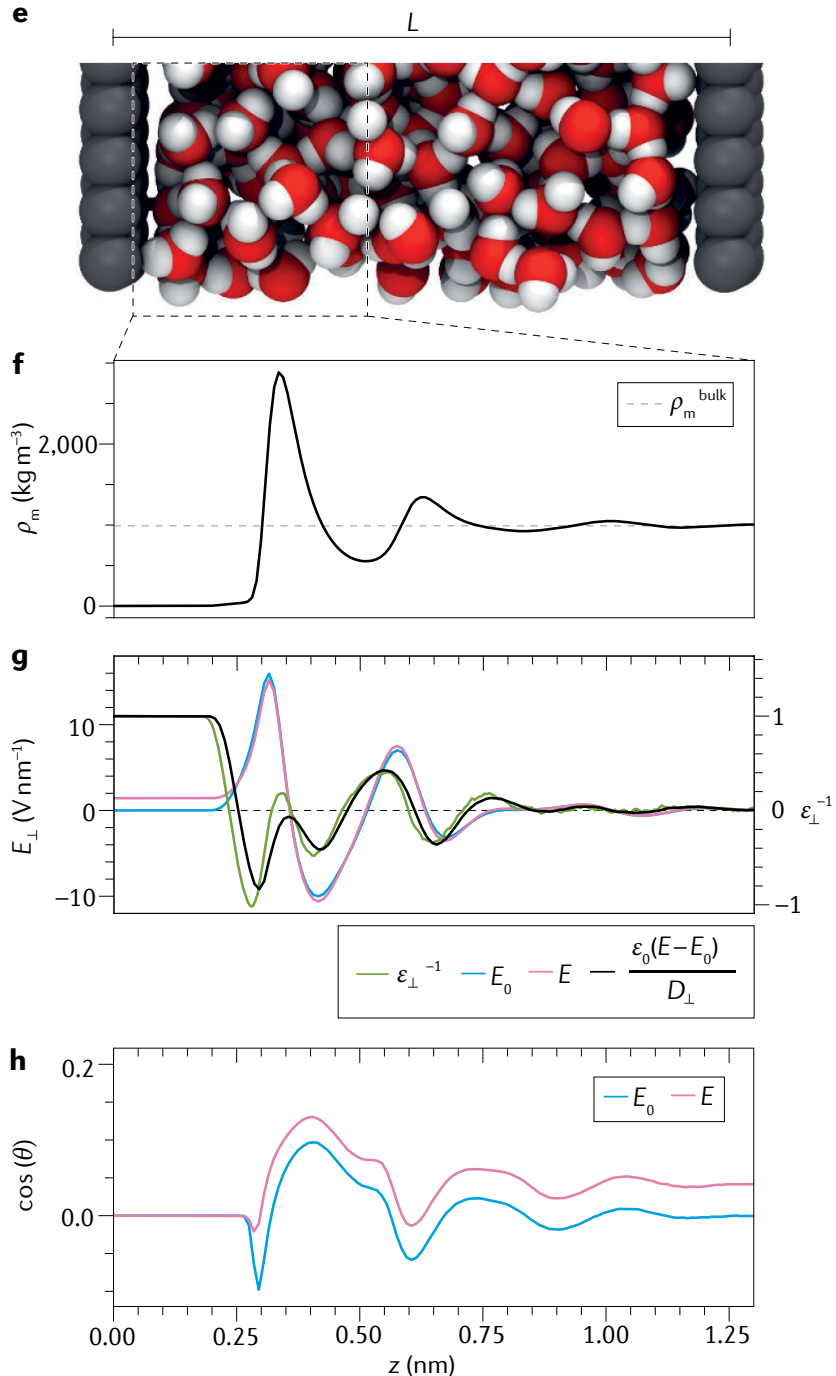

which changes insignificantly with increasing surface charge density. e | Molecular dynamics simulation snapshot for water between two flat neutral graphene surfaces. $\mathbf{f} \mid$ Pronounced water layering at the graphene surface is indicated by oscillations of the water mass density profile. g | Comparison of the intrinsic electric field profile $E_{0}$ (blue line) in the absence of an external field that is solely due to water orientation, and the electric field profile $E$ (pink line) in the presence of an externally applied constant displacement field $D / \varepsilon_{0}=1 \mathrm{Vnm}^{-1}$ that corresponds to a moderate surface charge of about $\sigma=+0.1 \mathrm{enm}^{-2}$. The inverse dielectric profile from the difference of the $E$ and $E_{0}$ fields (black line) compares well to the linear-response prediction (green line), which indicates that dielectric linear response is still valid. $\mathbf{h} \mid$ Water orientation profiles for $D / \varepsilon_{0}=0$ (blue line) and $D / \varepsilon_{0}=1 \mathrm{Vnm}^{-1}$ (pink line), which are related to the electric field profiles, indicate water reorientation induced by the externally applied displacement field. PB, Poisson-Boltzmann. Panels a-d adapted with permission from REF. ${ }^{178}$, American Chemical Society. Parts $\mathbf{e}-\mathbf{g}$ adapted with permission from REF. ${ }^{198}$, American Chemical Society. Part $\mathbf{h}$ is obtained from data reported in REFS ${ }^{197,198}$. re-formation of adsorbed $\mathrm{H}_{2} \mathrm{O}$ unlikely. So, varying the $\mathrm{pH}$ provides a means to control the molecular versus dissociative water interaction with anatase surfaces, as also reported for rutile ${ }^{221,222}$. These results are in good agreement with a combined SFG and ab initio MD study of water in contact with a film of anatase $\mathrm{TiO}_{2}$ particles, where chemisorbed water, forming hydroxyls group, and physisorbed water, strongly hydrogen-bonded to the oxygen of the hydroxyls groups, have been observed ${ }^{59}$.

On metal surfaces, electrochemical energy conversion occurs through interfacial charge transfer reactions, the mechanism and rate of which are mainly governed by the structure of the boundary region between the electronic (electrode) and ionic (electrolyte) conductors ${ }^{25}$. 
To fully understand hydration processes and the underlying chemical interactions, it is, therefore, helpful to experimentally access the electronic structure of the aqueous phase-charged electrode interface. Here, valence electrons of water and embedded solutes, at and near the immediate interface, play a key role because their energies govern chemical reactions. This includes electronic energy and charge transfer, proton transfer and the solution's influence on bonding and antibonding states that determine covalent interactions.

Not only does the electrode potential affect the structure of water near the surface but, because water can decompose to form adsorbed hydrogen and hydroxide, transferring electrons to or from the electrode surface, the variation of the electrode potential will favour the adsorption of these species ${ }^{202}$. As intermediates in various important reactions and potential site-blocking spectators for any reaction carried out in an aqueous environment ${ }^{223}$, understanding the thermodynamics and kinetics of their adsorption is important. The adsorption of the products of water decomposition $\left(\mathrm{H}^{*}, \mathrm{OH}^{*}\right.$ and $\left.\mathrm{O}^{*}\right)$ depends on the electrode potential, composition and surface structure ${ }^{209-212}$. A combination of detailed experiments on single-crystal platinum electrodes and density functional theory (DFT) calculations show that adsorption of all these species is more favourable onto less coordinated and, therefore, more strongly binding atoms at step edges ${ }^{224,225}$ compared with those on terraces ${ }^{226-228}$. As hydroxide ions contain an internal dipole, their adsorption favours the co-adsorption of water, to which the hydroxide strongly hydrogen bonds $\mathrm{s}^{229,230}$ and favours adsorption of water ${ }^{231}$. As the surface structure affects the length and angle of the hydrogen bonds formed, the effect of water on $\mathrm{OH}^{*}$ adsorption is surface-structure-dependent $t^{227,231}$. The binding strength of hydroxide also depends on the cation and $\mathrm{pH}$, because ions close to the surface disrupt the strong hydrogen bonds between $\mathrm{OH}^{*}$ and $\mathrm{H}_{2} \mathrm{O}^{*}$ $\left(\operatorname{REFS}^{227,228,231,232}\right)$. These effects are illustrated in FIG. 5.

The structure of interfacial water has been considered particularly important for the hydrogen oxidation reaction and the hydrogen evolution reaction (HER) in hydrogen fuel cells and electrolysers. In particular, the effect of $\mathrm{pH}$ and electrolyte ions has been substantially studied. It has been observed that the rates of the hydrogen oxidation reaction and the HER are 2-3 orders of magnitude slower in an alkaline electrolyte than in an acid electrolyte, even in the presence of the best catalysts known for this reaction, for example, platinum ${ }^{233-235}$. Recent experiments have shown that lowering the electrode $E_{\mathrm{pzc}}$ through adsorption of $\mathrm{Ni}(\mathrm{OH})_{2}$ increases the activity for the $\mathrm{HER}^{123,236}$. At a given potential, lowering of the $E_{\mathrm{pzc}}$ (caused by the presence of $\mathrm{Ni}(\mathrm{OH})_{2}$ ) leads to a decrease in the electric field strength across the electrode-electrolyte interface, as compared with the bare surface without $\mathrm{Ni}(\mathrm{OH})_{2}$ at that same potential. This decrease in field putatively reduces the energy required to reorient water near the electrode surface during adsorption of a proton, an important step in the HER, explaining the increased activity. If true, this suggests that the decreased activity seen with increasing $\mathrm{pH}$ could be caused by the larger electric field established at the same potential on a relative hydrogen (proton chemical potential) electrode scale with increasing $\mathrm{pH}$ (as the point of zero charge is $\mathrm{pH}$-independent) ${ }^{237}$.

\section{Outlook}

In this Review, we have discussed how charged interfaces of different nature induce the formation of the EDL in the presence of an aqueous electrolyte solution and affect the interfacial water structure. We have also given a brief introduction to the classical mean-field models and shown their limitations for the description of each component, namely, the charged surface, water and ions, in real systems of interest. In particular, we have focused on phenomena unique to non-ideal interfaces, such as those of biomembranes and minerals, and chemical reactions occurring at the metal-water or mineralwater interfaces. Classical theories can describe these systems only to a limited extent, as many phenomena violate the assumptions that these models rely on. These assumptions are (1) the surface is laterally homogeneous and charge distributions can be described by their spatial average in the plane of the interface (there are no domains or charged patches); (2) the charged surface is perfectly sharp; (3) ions only interact through Coulombic interactions; (4) there are no ion-ion correlations; and (5) water is a homogeneous dielectric continuum. Remarkably enough, these standard continuum models break down even for low-concentration electrolyte and at much larger length scales than molecular ones - situations for which one would expect that they would work. These theories provide a generally useful framework, but deviations from ideal behaviour assumed in these formalisms often become relevant for the description of systems discussed in this Review.

Mean-field theories have been improved by adding microscopic details such as the interfacial water properties and specific ion interactions. Yet, even these refined mean-field approaches ${ }^{178,204-208}$ are often not sufficient to get a full molecular picture. MD simulations, on the other hand, are plagued by force field and computing time issues. Because most force field models have been designed to accurately describe water-ion interactions, they often do not describe ion-ion interactions accurately. However, ion-ion interactions become increasingly important for the structure and dynamics of the whole system as the ion concentration increases. Thus, developing universal force field models for highly concentrated ion solutions is needed, as recently demonstrated for $\mathrm{Mg}^{2+}$ and $\mathrm{SO}_{4}{ }^{2-}$ ions ${ }^{238}$. An alternative route, which emerged in the past 10 years thanks to the development of methods that drastically reduce the computational effort, is using DFT-based molecular dynamics (DFT-MD) simulations. This approach offers a realistic representation of the electronic, structural and dynamic properties, and can provide a consistent atomistic description of the system ${ }^{34}$. DFT-MD has proven successful in describing many different observables, among which are the structure of adsorbed water molecules at solid interfaces, the surface acidity, the redox potential, the dielectric constant of polar liquids and the capacitance of the Stern layer at charged oxide-electrolyte interfaces, and hold promise for further extension 
to, among others, the modelling of metal-electrolyte interfaces (see REF. ${ }^{34}$ and references therein).

From the experimental point of view, ideally, one could make a movie, with molecular temporal and spatial resolution, of water and ions at charged interfaces. This requires combining ultrashort laser pulses with scanning probe microscopy ${ }^{239}$ and/or triggering charge transfer at an interface using ultrashort pulses and monitoring the temporal evolution of the system spectroscopically ${ }^{240}$. Such experiments can also help investigate systems out of equilibrium. So far, we have discussed the organization of ions and water, and chemical reactions at charged surfaces under equilibrium conditions. However, the majority of important systems and applications function under a non-equilibrium but steady-state situation. For example, living systems exist because of their ability to maintain non-equilibrium conditions across their membranes; water flowing along mineral rocks, shifting dissolution equilibria and, thereby, charge ${ }^{10}$; at the battery electrode-electrolyte interface, electrons are continually converted into ions, and hydration is continuously changing. Along with this chemical conversion, diffusion and reorientation of chemical species happen. Such interfacial non-equilibrium dynamics are central to many important applications. Molecular-level details of water at charged interfaces remain poorly understood for equilibrated systems, but even more so for non-equilibrium states. A key question that needs to be addressed is to what extent the modern EDL theories work or break down under non-equilibrium conditions. To address this, a combination of in situ, operando, time-resolved and surface-specific measurement, together with simulations using non-equilibrium techniques in the grand canonical ensemble, are needed. Thus, the road ahead is paved by big challenges, yet, if successful, the reward will be great, by allowing fundamental scientific insights to have a concrete impact in more applied fields.

Published online 24 June 2021
1. Hurd C. L., Lenton, A, Tilbrook, B. \& Boyd, P. W. Current understanding and challenges for oceans in a higher- $\mathrm{CO}_{2}$ world. Nat. Clim. Change 8, 686-694 (2018).

2. Putnis, A. Why mineral interfaces matter. Science 343, 1441-1442 (2014)

3. Casey, W. H., Rustad, J. R. \& Spiccia, L. Minerals as molecules - Use of aqueous oxide and hydroxide clusters to understand geochemical reactions. Chem. Eur. J. 15, 4496-4515 (2009).

4. Villa, E. M., Ohlin, C. A. \& Casey, W. H. Oxygenisotope exchange rates for three isostructural polyoxometalate ions. J. Am. Chem. Soc. 132 5264-5272 (2010)

5. Lipfert, J., Doniach, S., Das, R. \& Herschlag, D. Understanding nucleic acid-ion interactions. Annu. Rev. Biochem. 83, 813-841 (2014).

6. Laage, D., Elsaesser, T. \& Hynes, J. T. Water dynamics in the hydration shells of biomolecules. Chem. Rev. 117, 10694-10725 (2017).

7. Zhou, H.-X. \& Pang, X. Electrostatic interactions in protein structure, folding, binding, and condensation. Chem. Rev. 118, 1691-1741 (2018).

8. Agmon, N. et al. Protons and hydroxide ions in aqueous systems. Chem. Rev. 116, 7642-7672 (2016).

9. Teissie, J., Prats, M., Soucaille, P. \& Tocanne, J. F. Evidence for conduction of protons along the interface between water and a polar lipid monolayer. Proc. Natl Acad. Sci. USA 82, 3217-3221 (1985).

10. Williams, R. J. P. Proton circuits in biological energy interconversions. Annu. Rev. Biophys. Biophys. Chem. 17, 71-97 (1988).

11. Heberle, J., Riesle, J., Thiedemann, G., Oesterhelt, D. \& Dencher, N. A. Proton migration along the membrane surface and retarded surface to bulk transfer. Nature 370, 379-382 (1994).

12. Sandén, T., Salomonsson, L., Brzezinski, P. \& Widengren, J. Surface-coupled proton exchange of a membrane-bound proton acceptor. Proc. Natl Acad. Sci. USA 107, 4129-4134 (2010).

13. Zhang, $\mathrm{C}$. et al. Water at hydrophobic interfaces delays proton surface-to-bulk transfer and provides a pathway for lateral proton diffusion. Proc. Natl Acad. Sci. USA 109, 9744-9749 (2012).

14. Amdursky, N., Lin, Y., Aho, N. \& Groenhof, G. Exploring fast proton transfer events associated with lateral proton diffusion on the surface of membranes. Proc. Natl Acad. Sci. USA 116, 2443-2451 (2019).

15. Kundu, A. et al. Hydrated excess protons in acetonitrile/water mixtures: solvation species and ultrafast proton motions. J. Phys. Chem. Lett. 10, 2287-2294 (2019)

16. Fujishima, A. \& Honda, K. Electrochemical photolysis of water at a semiconductor electrode. Nature $\mathbf{2 3 8}$, 37-38 (1972)

17. Walter, M. G. et al. Solar water splitting cells Chem. Rev. 110, 6446-6473 (2010).

18. Maeda, K. Photocatalytic water splitting using semiconductor particles: history and recent developments J. Photochem Photobiol. C Photochem. Rev. 12, 237-268 (2011)

19. Osterloh, F. E. Inorganic nanostructures for photoelectrochemical and photocatalytic water splitting. Chem. Soc. Rev. 42, 2294-2320 (2013).

20. Ran, J., Zhang, J., Yu, J., Jaroniec, M. \& Qiao, S. Z Earth-abundant cocatalysts for semiconductor-based photocatalytic water splitting. Chem. Soc. Rev. 43, 7787-7812 (2014).

21. Ormerod, R. M. Solid oxide fuel cells. Chem. Soc. Rev. 32, 17-28 (2003).

22. Wachsman, E. D. \& Lee, K. T. Lowering the temperature of solid oxide fuel cells. Science 334 935-939 (2011)

23. Debe, M. K. Electrocatalyst approaches and challenges for automotive fuel cells. Nature $\mathbf{4 8 6}$ 43-51 (2012).

24. Park, S., Shao, Y., Liu, J. \& Wang, Y. Oxygen electrocatalysts for water electrolyzers and reversible fuel cells: status and perspective. Energy Environ. Sci. 5, 9331-9344 (2012).

25. Trasatti, S. Surface science and electrochemistry: concepts and problems. Surf. Sci. 335, 1-9 (1995).

26. Guo, Y.-G., Hu, J.-S. \& Wan, L.-J. Nanostructured materials for electrochemical energy conversion and storage devices. Adv. Mater. 20, 2878-2887 (2008)

27. Hou, J., Shao, Y., Ellis, M. W., Moore, R. B. \& Yi, B. Graphene-based electrochemical energy conversion and storage: fuel cells, supercapacitors and lithium ion batteries. Phys. Chem. Chem. Phys. 13 15384-15402 (2011)

28. Zhang, X., Cheng, X. \& Zhang, Q. Nanostructured energy materials for electrochemical energy conversion and storage: a review. J. Energy Chem 25, 967-984 (2016).

29. Bonn, M., Nagata, Y. \& Backus, E. H. G. Molecular structure and dynamics of water at the water-air interface studied with surface-specific vibrational spectroscopy. Angew. Chem. Int. Ed. 54, 5560-5576 (2015).

30. Maier, S. \& Salmeron, M. How does water wet a surface? Acc. Chem. Res. 48, 2783-2790 (2015).

31. Pekker, M. \& Shnelder, M. N. Interaction between electrolyte ions and the surface of a cell lipid membrane. J. Phys. Chem. Biophys. 5, 177 (2015).

32. Björneholm, O. et al. Water at interfaces. Chem. Rev. 116, 7698-7726 (2016)

33. Gro $\beta, A$. in Surface and Interface Science: Interfacial Electrochemistry 1st edn (ed. Wandelt, K.) 471-515 (Wiley, 2020).

34. Zhang, C., Sayer, T., Hutter, J. \& Sprik, M. Modelling electrochemical systems with finite field molecular dynamics. J. Phys. Energy 2, 032005 (2020).

35. Backus, E. H. G., Schaefer, J. \& Bonn, M Probing the mineral-water interface with nonlinear optical spectroscopy. Angew. Chem. Int. Ed. 60 10482-10501 (2021).

36. Kučerka, N., Gallová, J. ¿ Uhríková, D. The membrane structure and function affected by water. Chem. Phys. Lipids 221, 140-144 (2019).
37. Okur, H. I., Tarun, O. B. \& Roke, S. Chemistry of lipid membranes from models to living systems: a perspective of hydration, surface potential, curvature, confinement and heterogeneity. J. Am. Chem. Soc. 141, 12168-12181 (2019).

38. Garrett, B. C. et al. Role of water in electron-initiated processes and radical chemistry: issues and scientific advances. Chem. Rev. 105, 355-389 (2005).

39. Nagata, Y., Ohto, T., Backus, E. H. G. \& Bonn, M. Molecular modeling of water interfaces: from molecular spectroscopy to thermodynamics. J. Phys. Chem. B 120, 3785-3796 (2016).

40. Striolo, A. Interfacial water studies and their relevance for the energy sector. Mol. Phys. 114, 2615-2626 (2016).

41. Pasenkiewicz-Gierula, M., Baczynski, K., Markiewicz, M. $\&$ Murzyn, K. Computer modelling studies of the bilayer/water interface. Biochim. Biophys. Acta Biomembr. 1858, 2305-2321 (2016).

42. Helmholtz, H. Ueber einige Gesetze der Vertheilung elektrischer Ströme in körperlichen Leitern mit Anwendung auf die thierisch-elektrischen Versuche. Ann. Phys. Chem. 89, 211-233 (1853).

43. Helmholtz, H. Studien über electrische Grenzschichten Ann. Phys. Chem. 243, 337-382 (1879).

44. Gouy, M. Sur la constitution de la charge électrique à la surface d'un électrolyte. J. Phys. Theor. Appl. 9, 457-468 (1910)

45. Chapman, D. L. A contribution to the theory of electrocapillarity. London Edinburgh Dublin Philos. Mag. J. Sci. 25, 475-481 (1913).

46. Debye, P. \& Hückel, E. Zur Theorie der Elektrolyte. I. Gefrierpunktserniedrigung und verwandte Erscheinungen. Phys. Z. 24, 185-206 (1923).

47. Stern, O. Zur theorie der elektrolytischen doppelschicht. Z. Elektrochem. Angew. Phys. Chem. 30, 508-516 (1924).

48. Grahame, D. C. The electrical double layer and the theory of electrocapillarity. Chem. Rev. 41, 441-501 (1947).

49. Conway, B. E., Bockris, J. O. M. \& Ammar, I. A The dielectric constant of the solution in the diffuse and Helmholtz double layers at a charged interface in aqueous solution. Trans. Faraday Soc. 47, 756-766 (1951).

50. Bockris, J O. Devanathan, M. A V. \& Müller, K. On the structure of charged interfaces. Proc. R. Soc. Lond. A Math. Phys. Sci. 274, 55-79 (1963).

51. Verwey, E. J. W. \& Overbeek, J. T. G. Theory of the Stability of Lyophobic Colloids (Elsevier, 1948)

52. Uematsu, Y., Netz, R. R. \& Bonthuis, D. J. Analytical interfacial layer model for the capacitance and electrokinetics of charged aqueous interfaces. Langmuir 34, 9097-9113 (2018).

53. Bard, A. J. \& Faulkner, L. R. Electrochemical Methods: Fundamentals and Applications 2nd edn (Wiley, 2000).

54. Bockris, J. O. \& Reddy, A. K. N. The electrified interface. in Modern Electrochemistry: An Introduction to an Interdisciplinary Area Vol. 2 623-843 (Springer, 1970). 
55. Fumagalli, L. et al. Anomalously low dielectric constant of confined water. Science 360, 1339-1342 (2018)

56. Winiski, A. P., McLaughlin, A. C., McDaniel, R. V., Eisenberg, M. \& McLaughlin, S. An experimental test of the discreteness-of-charge effect in positive and negative lipid bilayers. Biochemistry 25, 8206-8214 (1986).

57. Israelachvili, J. N. \& Adams, G. E. Measurement of forces between two mica surfaces in aqueous electrolyte solutions in the range $0-100 \mathrm{~nm}$. J. Chem. Soc. Faraday Trans. 1 Phys. Chem. Condens. Phases 74, 975-1001 (1978).

58. Langner, M., Cafiso, D., Marcelja, S. \& McLaughlin, S. Electrostatics of phosphoinositide bilayer membranes. Theoretical and experimental results. Biophys. J. 57, 335-349 (1990).

59. Hosseinpour, S. et al. Chemisorbed and physisorbed water at the $\mathrm{TiO}_{2} /$ water interface. J. Phys. Chem. Lett. 8, 2195-2199 (2017)

60. Ali, H., Seidel, R., Bergmann, A. \& Winter, B. Electronic structure of aqueous-phase anatase titanium dioxide nanoparticles probed by liquid jet photoelectron spectroscopy. J. Mater. Chem. A 7, 6665-6675 (2019)

61. Gallagher, F. A. et al. Magnetic resonance imaging of $\mathrm{pH}$ in vivo using hyperpolarized ${ }^{13} \mathrm{C}$-labelled bicarbonate. Nature 453, 940-943 (2008)

62. Zhang, X., Lin, Y. \& Gillies, R. J. Tumor $\mathrm{pH}$ and its measurement. J. Nucl. Med. 51, 1167-1170 (2010).

63. Casey, J. R., Grinstein, S. \& Orlowski, J. Sensors and regulators of intracellular pH. Nat. Rev. Mol. Cell Biol. 11, 50-61 (2009).

64. Cevc, G. Phospholipids Handbook (CRC Press, 2018).

65. Alberts, B. et al. Molecular Biology of the Cell (Garland Science, 2013).

66. Petrache, H. I., Zemb, T., Belloni, L. \& Parsegian, V. A. Salt screening and specific ion adsorption determine neutral-lipid membrane interactions. Proc. Natl Acad. Sci. USA 103, 7982-7987 (2006).

67. Dreier, L. B. et al. Unraveling the origin of the apparent charge of zwitterionic lipid layers. J. Phys. Chem. Lett. 10, 6355-6359 (2019)

68. Yamaguchi, S., Bhattacharyya, K. \& Tahara, T Acid-base equilibrium at an aqueous interface: $\mathrm{pH}$ spectrometry by heterodyne-detected electronic sum frequency generation. J. Phys. Chem. C 115, 4168-4173 (2011)

69. Kundu, A., Yamaguchi, S. \& Tahara, T. Evaluation of $\mathrm{pH}$ at charged lipid/water interfaces by heterodyne detected electronic sum frequency generation. J. Phys. Chem. Lett. 5, 762-766 (2014).

70. Zhang, T., Cathcart, M. G., Vidalis, A. S. \& Allen, H. C. Cation effects on phosphatidic acid monolayers at various $\mathrm{pH}$ conditions. Chem. Phys. Lipids $\mathbf{2 0 0}$ 24-31 (2016).

71. Zhang, T. et al. Effect of $\mathrm{pH}$ and salt on surface $\mathrm{pKa}$ of phosphatidic acid monolayers. Langmuir 34 530-539 (2018)

72. Tsui, F. C., Ojcius, D. M. \& Hubbell, W. L. The intrinsic pKa values for phosphatidylserine and phosphatidylethanolamine in phosphatidylcholine host bilayers. Biophys. J. 49, 459-468 (1986).

73. Melcrová, A., Pokorna, S., Pullanchery, S. ¿ Kohagen, M The complex nature of calcium cation interactions with phospholipid bilayers. Sci. Rep. 6, 38035 (2016).

74. Lis, D., Backus, E. H. G., Hunger, J., Parekh, S. H. \& Bonn, M. Liquid flow along a solid surface reversibly alters interfacial chemistry. Science 344, 1138-1142 (2014).

75. Khatib, R. et al. Water orientation and hydrogen-bond structure at the fluorite/water interface. Sci. Rep. 6 24287 (2016).

76. Sulpizi, M., Gaigeot, M. P. \& Sprik, M. The silicawater interface: how the silanols determine the surface acidity and modulate the water properties. J. Chem. Theory Comput. 8, 1037-1047 (2012).

77. Iler, R. K. The Chemistry of Silica: Solubility, Polymerization, Colloid and Surface Properties and Biochemistry of Silica (Wiley, 1979)

78. Leung, K., Nielsen, I. M. B. \& Criscenti, L. J. Elucidating the bimodal acid-base behavior of the water-silica interface from first principles. J. Am. Chem. Soc. 131, 18358-18365 (2009).

79. Lee, S. S., Fenter, P., Nagy, K. L. \& Sturchio, N. C. Real-time observation of cation exchange kinetics and dynamics at the muscovite-water interface. Nat. Commun. 8, 15826 (2017).

80. Ong, S., Zhao, X. \& Eisenthal, K. B. Polarization of water molecules at a charged interface: second harmonic studies of the silica/water interface. Chem. Phys. Lett. 191, 327-335 (1992).
81. Evans, D. F. \& Wennerström, H. The Colloidal Domain: Where Physics, Chemistry, Biology, and Technology Meet. Advances in Interfacial Engineering (Wiley, 1999).

82. Wen, Y.-C. et al. Unveiling microscopic structures of charged water interfaces by surface-specific vibrational spectroscopy. Phys. Rev. Lett. 116, 16101 (2016).

83. Schaefer, J., Gonella, G., Bonn, M. \& Backus, E. H. G. Surface-specific vibrational spectroscopy of the water/silica interface: screening and interference. Phys. Chem. Chem. Phys. 19, 16875-16880 (2017)

84. Hore, D. K. \& Tyrode, E. Probing charged aqueous interfaces near critical angles: effect of varying coherence length. J. Phys. Chem. C 123 16911-16920 (2019).

85. Zhang, Y. \& Cremer, P. S. Chemistry of Hofmeister anions and osmolytes. Annu. Rev. Phys. Chem. 61 63-83 (2010).

86. Govrin, R., Schlesinger, I., Tcherner, S. \& Sivan, U. Regulation of surface charge by biological osmolytes. J. Am. Chem. Soc. 139, 15013-15021 (2017).

87. Jan Akhunzada, M et al. Interplay between lipid lateral diffusion, dye concentration and membrane permeability unveiled by a combined spectroscopic and computational study of a model lipid bilayer. Sci. Rep. 9, 1508 (2019).

88. Tarun, O. B., Hannesschläger, C., Pohl, P. \& Roke, S. Label-free and charge-sensitive dynamic imaging of lipid membrane hydration on millisecond time scales. Proc. Natl Acad. Sci. USA 115, 4081-4086 (2018).

89. Cyran, J. D. et al. Molecular hydrophobicity at a macroscopically hydrophilic surface. Proc. Natl Acad. Sci. USA 116, 1520-1525 (2019).

90. Macias-romero, C., Nahalka, I., Okur, H. I. \& Roke, S. Optical imaging of surface chemistry and dynamics in confinement. Science 357, 784-788 (2017).

91. Favaro, M. et al. Unravelling the electrochemical double layer by direct probing of the solid/liquid interface. Nat. Commun. 7, 12695 (2016).

92. Smith, A. M., Lee, A. A. \& Perkin, S. The electrostatic screening length in concentrated electrolytes increases with concentration. J. Phys. Chem. Lett. 7 2157-2163 (2016).

93. Ojha, K., Arulmozhi, N., Aranzales, D. \& Koper, M. T. M. Double layer at the Pt(111)-aqueous electrolyte interface: potential of zero charge and anomalous Gouy-Chapman screening. Angew. Chem. Int. Ed. 59 , 711-715 (2020)

94. Hofmeister, F. Zur Lehre von der Wirkung der Salze. Arch. Exp. Pathol. Pharmacol. 24, 247-260 (1888).

95. Collins, K. D. Ions from the Hofmeister series and osmolytes: effects on proteins in solution and in the crystallization process. Methods 34, 300-311 (2004).

96. Schwierz, N., Horinek, D. \& Netz, R. R. Anionic and cationic Hofmeister effects on hydrophobic and hydrophilic surfaces. Langmuir 29, 2602-2614 (2013).

97. Morag, J., Dishon, M. \& Sivan, U. The governing role of surface hydration in ion specific adsorption to silica: an AFM-based account of the Hofmeister universality and its reversal. Langmuir 29, 6317-6322 (2013).

98. Kunz, W., Lo Nostro, P. \& Ninham, B. W. The present state of affairs with Hofmeister effects. Curr. Opin. Colloid Interface Sci. 9, 1-18 (2004).

99. Gopalakrishnan, S., Liu, D., Allen, H. C., Kuo, M. \& Shultz, M. J. Vibrational spectroscopic studies of aqueous interfaces: salts, acids, bases, and nanodrops. Chem. Rev. 106, 1155-1175 (2006).

100. Pegram, L. M. \& Record, M. T. Thermodynamic origin of Hofmeister ion effects. J. Phys. Chem. B 112 9428-9436 (2008).

101. Lyklema, J. Quest for ion-ion correlations in electric double layers and overcharging phenomena. $A d v$. Colloid Interface Sci. 147-148, 205-213 (2009).

102. Allen, H. C., Casillas-Ituarte, N. N., Sierra-Hernández, M. R., Chen, X. \& Tang, C. Y. Shedding light on water structure at air-aqueous interfaces: ions, lipids, and hydration. Phys. Chem. Chem. Phys. 11, 5538-5549 (2009).

103. Tang, C. Y. \& Allen, H. C. Ionic binding of $\mathrm{Na}^{+}$versus $\mathrm{K}$ to the carboxylic acid headgroup of palmitic acid monolayers studied by vibrational sum frequency generation spectroscopy. J. Phys. Chem. A 113 7383-7393 (2009).

104. Casillas-Ituarte, N. N., Chen, X., Castada, H. \& Allen, H.C. $\mathrm{Na}^{+}$and $\mathrm{Ca}^{2+}$ effect on the hydration and orientation of the phosphate group of DPPC at air-water and air-hydrated silica interfaces. J. Phys. Chem. B 114, 9485-9495 (2010).

105. Schwierz, N., Horinek, D. \& Netz, R. R. Reversed anionic Hofmeister series: the interplay of surface charge and surface polarity. Langmuir 26, 7370-7379 (2010).
106. Jungwirth, P. Spiers Memorial Lecture: Ions at aqueous interfaces. Faraday Discuss. 141, 9-30 (2008).

107. Macdonald, P. M. \& Seelig, J. Anion binding to neutral and positively charged lipid membranes. Biochemistry 27, 6769-6775 (1988).

108. Gurtovenko, A. A., Miettinen, M., Karttunen, M. \& Vattulainen, I. Effect of monovalent salt on cationic lipid membranes as revealed by molecular dynamics simulations. J. Phys. Chem. B 109, 21126-21134 (2005).

109. Garcia-Celma, J. J., Hatahet, L., Kunz, W. \& Fendler, K. Specific anion and cation binding to lipid membranes investigated on a solid supported membrane. Langmuir 23, 10074-10080 (2007).

110. Vácha, R. et al. Effects of alkali cations and halide anions on the DOPC lipid membrane. J. Phys. Chem. A 113, 7235-7243 (2009).

111. Jurkiewicz, P., Cwiklik, L., Vojtǐšková, A., Jungwirth, P. $\&$ Hof, M. Structure, dynamics, and hydration of POPC/POPS bilayers suspended in $\mathrm{NaCl}, \mathrm{KCl}$, and $\mathrm{CsCl}$ solutions. Biochim. Biophys. Acta Biomembr. 1818, 609-616 (2012)

112. Pokorna, S. et al. Does fluoride disrupt hydrogen bond network in cationic lipid bilayer? Time-dependent fluorescence shift of Laurdan and molecular dynamics simulations. J. Chem. Phys. 141, 22D516 (2014).

113. Melcr, J. et al. Accurate binding of sodium and calcium to a POPC bilayer by effective inclusion of electronic polarization. J. Phys. Chem. B 122, 4546-4557 (2018).

114. Yang, Z., Li, Q. \& Chou, K. C. Structures of water molecules at the interfaces of aqueous salt solutions and silica: cation effects. J. Phys. Chem. C 113, 8201-8205 (2009).

115. Flores, S. C., Kherb, J., Konelick, N., Chen, X. \& Cremer, P. S. The effects of Hofmeister cations at negatively charged hydrophilic surfaces. J. Phys. Chem. C 116, 5730-5734 (2012).

116. Azam, M. S., Weeraman, C. N. \& Gibbs-Davis, J. M Specific cation effects on the bimodal acid-base behavior of the silica/water interface. J. Phys. Chem Lett. 3, 1269-1274 (2012).

117. Azam, M. S., Weeraman, C. N. \& Gibbs-Davis, J. M. Halide-induced cooperative acid-base behavior at a negatively charged interface. J. Phys. Chem. C 117, 8840-8850 (2013).

118. Dewan, S. et al. Structure of water at charged interfaces: a molecular dynamics study. Langmuir 30 , 8056-8065 (2014).

119. Lovering, K. A., Bertram, A. K. \& Chou, K. C. New information on the ion-identity-dependent structure of Stern layer revealed by sum frequency generation vibrational spectroscopy. J. Phys. Chem. C 120 18099-18104 (2016)

120. DeWalt-Kerian, E. L. et al. pH-Dependent inversion of Hofmeister trends in the water structure of the electrical double layer. J. Phys. Chem. Lett. 8 , 2855-2861 (2017).

121. Valette, G. Double layer on silver single-crystal electrodes in contact with electrolytes having anions which present a slight specific adsorption. Part I. The (110) face. J. Electroanal. Chem. 122, 285-297 (1981).

122. Thorson, M. R., Siil, K. I. \& Kenis, P. J. A. Effect of cations on the electrochemical conversion of $\mathrm{CO}_{2}$ to CO. J. Electrochem. Soc. 160, F69-F74 (2013).

123. Ledezma-Yanez, I. et al. Interfacial water reorganization as a $\mathrm{pH}$-dependent descriptor of the hydrogen evolution rate on platinum electrodes. Nat. Energy 2, 17031 (2017).

124. Resasco, J. et al. Promoter effects of alkali metal cations on the electrochemical reduction of carbon dioxide. J. Am. Chem. Soc. 139, 11277-11287 (2017).

125. Liu, X. et al. pH effects on the electrochemical reduction of $\mathrm{CO}_{(2)}$ towards $\mathrm{C}_{2}$ products on stepped copper. Nat. Commun. 10, 32 (2019).

126. Ringe, S. et al. Understanding cation effects in electrochemical $\mathrm{CO}_{2}$ reduction. Energy Environ. Sci. 12, 3001-3014 (2019)

127. Collins, K. D., Neilson, G. W. \& Enderby, J. E. Ions in water: characterizing the forces that control chemical processes and biological structure. Biophys. Chem. 128, 95-104 (2007)

128. Vlachy, N. et al. Hofmeister series and specific interactions of charged headgroups with aqueous ions. Adv. Colloid Interface Sci. 146, 42-47 (2009).

129. James, R. O. \& Healy, T. W. Adsorption of hydrolyzable metal ions at the oxide-water interface. II. Charge reversal of $\mathrm{SiO}_{2}$ and $\mathrm{TiO}_{2}$ colloids by adsorbed $\mathrm{Co}(\mathrm{II})$, $\mathrm{La}(\mathrm{III})$, and Th(IV) as model systems. J. Colloid Interface Sci. 40, 53-64 (1972). 
130. Levin, Y. Electrostatic correlations: from plasma to biology. Rep. Prog. Phys. 65, 1577-1632 (2002).

131. Linse, P. Mean force between like-charged macroions at high electrostatic coupling. J. Phys. Condens. Matter 14, 13449-13467 (2002).

132. Jönsson, B. \& Wennerström, H. Ion-ion correlations in liquid dispersions. J. Adhes. 80, 339-364 (2004).

133. Boroudjerdi, H. et al. Statics and dynamics of strongly charged soft matter. Phys. Rep. 416, 129-199 (2005).

134. Pegado, L., Jönsson, B. \& Wennerström, H. Attractive ion-ion correlation forces and the dielectric approximation. Adv. Colloid Interface Sci. 232, 1-8 (2016)

135. de Vos, W. M. \& Lindhoud, S. Overcharging and charge inversion: finding the correct explanation(s). Adv. Colloid Interface Sci. 274, 102040 (2019).

136. Guldbrand, L., Jönsson, B., Wennerström, H. \& Linse, P. Electrical double layer forces. A Monte Carlo study. J. Chem. Phys. 80, 2221-2228 (1984).

137. Khan, A., Joensson, B. \& Wennerstroem, H. Phase equilibria in the mixed sodium and calcium di-2-ethylhexylsulfosuccinate aqueous system. An illustration of repulsive and attractive double-layer forces. J. Phys. Chem. 89, 5180-5184 (1985).

138. Bratko, D., Jönsson, B. \& Wennerström, H. Electrical double layer interactions with image charges. Chem. Phys. Lett. 128, 449-454 (1986).

139. Rouzina, I. \& Bloomfield, V. A. Macroion attraction due to electrostatic correlation between screening counterions. 1. Mobile surface-adsorbed ions and diffuse ion cloud. J. Phys. Chem. 100, 9977-9989 (1996).

140. Netz, R. R. \& Joanny, J.-F. Adsorption of semiflexible polyelectrolytes on charged planar surfaces: charge compensation, charge reversal, and multilayer formation. Macromolecules 32, 9013-9025 (1999).

141. Shklovskii, B. I. Screening of a macroion by multivalent ions: correlation-induced inversion of charge. Phys. Rev. E 60, 5802-5811 (1999)

142. Moreira, A. G. \& Netz, R. R. Binding of similarly charged plates with counterions only. Phys. Rev. Lett. 87, 078301 (2001)

143. Grosberg, A. Y., Nguyen, T. T. \& Shklovskii, B. I. Colloquium: the physics of charge inversion in chemical and biological systems. Rev. Mod. Phys. 74, 329-345 (2002).

144. Dreier, L. B. et al. Saturation of charge-induced water alignment at model membrane surfaces. Sci. Adv. 4, eaap7415 (2018)

145. Brown, M. A. et al. Determination of surface potential and electrical double-layer structure at the aqueous electrolyte-nanoparticle interface. Phys. Rev. X 6 , 11007 (2016).

146. Lütgebaucks, C., Macias-Romero, C. \& Roke, S. Characterization of the interface of binary mixed DOPC:DOPS liposomes in water: the impact of charge condensation. J. Chem. Phys. 146, 044701 (2017).

147. Pullanchery, S., Yang, T. \& Cremer, P. S. Introduction of positive charges into zwitterionic phospholipid monolayers disrupts water structure whereas negative charges enhances it. J. Phys. Chem. B 122 12260-12270 (2018)

148. Manning, G. S. Limiting laws and counterion condensation in polyelectrolyte solutions I. Colligative properties. J. Chem. Phys. 51, 924-933 (1969).

149. Manning, G. S. The interaction between a charged wall and its counterions: a condensation theory. J. Phys. Chem. B 114, 5435-5440 (2010).

150. Gragson, D. E., McCarty, B. M. \& Richmond, G. L. Ordering of interfacial water molecules at the charged air/water interface observed by vibrational sum frequency generation. J. Am. Chem. Soc. 119 , 6144-6152 (1997).

151. Nilsson, A. \& Pettersson, L. G. M. The structural origin of anomalous properties of liquid water. Nat. Commun. 6, 8998 (2015)

152. Brini, E. et al. How water's properties are encoded in its molecular structure and energies. Chem. Rev. 117, 12385-12414 (2017).

153. Salmeron, M. et al. Water growth on metals and oxides: binding, dissociation and role of hydroxyl groups. Faraday Discuss. 141, 221-229 (2008)

154. Nihonyanagi, S., Yamaguchi, S. \& Tahara, T. Direct evidence for orientational flip-flop of water molecules at charged interfaces: a heterodyne-detected vibrational sum frequency generation study. J. Chem. Phys. 130, 204704 (2009).

155. Mondal, J. A., Nihonyanagi, S., Yamaguchi, S. \& Tahara, T. Structure and orientation of water at charged lipid monolayer/water interfaces probed by heterodyne-detected vibrational sum frequency generation spectroscopy. J. Am. Chem. Soc. 132 10656-10657 (2010)

156. Chen, X., Hua, W., Huang, Z. \& Allen, H. C. Interfacial water structure associated with phospholipid membranes studied by phase-sensitive vibrational sum frequency generation spectroscopy. J. Am. Chem. Soc. 132, 11336-11342 (2010).

157. Darlington, A. M. et al. Separating the $\mathrm{pH}$-dependent behavior of water in the Stern and diffuse layers with varying salt concentration. J. Phys. Chem. C 121 20229-20241 (2017).

158. Dutta, C., Mammetkuliyev, M. \& Benderskii, A. V. Re-orientation of water molecules in response to surface charge at surfactant interfaces. J. Chem. Phys. 151, 034703 (2019)

159. Rehl, B. et al. New Insights into $\chi^{(3)}$ measurements: comparing nonresonant second harmonic generation and resonant sum frequency generation at the silica/ aqueous electrolyte interface. J. Phys. Chem. C 123, 10991-11000 (2019).

160. Ostroverkhov, V., Waychunas, G. A. \& Shen, Y. R. Vibrational spectra of water at water/a-quartz $\left(\begin{array}{llll}0 & 0 & 0 & 1\end{array}\right)$ interface. Chem. Phys. Lett. 386, 144-148 (2004).

161. Darlington, A. M. \& Gibbs-Davis, J. M. Bimodal or trimodal? The influence of starting ph on site identity and distribution at the low salt aqueous/silica interface. J. Phys. Chem. C 119, 16560-16567 (2015).

162. Tielrooij, K. J., Van Der Post, S. T., Hunger, J., Bonn, M. \& Bakker, H. J. Anisotropic water reorientation around ions. J. Phys. Chem. B 115, 12638-12647 (2011).

163. Urashima, S. H., Myalitsin, A., Nihonyanagi, S. \& Tahara, T. The topmost water structure at a charged silica/aqueous interface revealed by heterodynedetected vibrational sum frequency generation spectroscopy. J. Phys. Chem. Lett. 9, 4109-4114 (2018).

164. Ahmed, M., Inoue, K., Nihonyanagi, S. \& Tahara, T. Hidden isolated $\mathrm{OH}$ at the charged hydrophobi interface revealed by two-dimensional heterodynedetected VSFG spectroscopy. Angew. Chem. Int. Ed. 59, 9498-9505 (2020)

165. Ohto, T. et al. Lipid carbonyl groups terminate the hydrogen bond network of membrane-bound water J. Phys. Chem. Lett. 6, 4499-4503 (2015).

166. Ishiyama, T., Terada, D. \& Morita, A. Hydrogenbonding structure at zwitterionic lipid/water interface. J. Phys. Chem. Lett. 7, 216-220 (2016).

167. Dreier, L. B., Bonn, M. \& Backus, E. H. G. Hydration and orientation of carbonyl groups in oppositely charged lipid monolayers on water. J. Phys. Chem. B 123, 1085-1089 (2019)

168. Marrink, S. J., Berkowitz, M. \& Berendsen, H. J. C. Molecular dynamics simulation of a membrane/water interface: the ordering of water and its relation to the hydration force. Langmuir 9, 3122-3131 (1993).

169. Mondal, J. A., Nihonyanagi, S., Yamaguchi, S. $\&$ Tahara, T. Three distinct water structures at a zwitterionic lipid/water interface revealed by heterodyne detected vibrational sum frequency generation. J. Am. Chem. Soc. 134, 7842-7850 (2012).

170. Lütgebaucks, C., Gonella, G. \& Roke, S. Optical labelfree and model-free probe of the surface potential of nanoscale and microscopic objects in aqueous solution. Phys. Rev. B 94, 195410 (2016).

171. Magarkar, A., Róg, T. \& Bunker, A. Molecular dynamics simulation of inverse-phosphocholine lipids. J. Phys. Chem. C 118, 19444-19449 (2014).

172. Toney, M. F. et al. Voltage-dependent ordering of water molecules at an electrode-electrolyte interface. Nature 368, 444-446 (1994).

173. García-Aráez, N., Climent, V. \& Feliu, J. M. Evidence of water reorientation on model electrocatalytic surfaces from nanosecond-laser-pulsed experiments. J. Am. Chem. Soc. 130, 3824-3833 (2008)

174. Velasco-Velez, J. J. et al. The structure of interfacial water on gold electrodes studied by $\mathrm{x}$-ray absorption spectroscopy. Science 346, 831-834 (2014).

175. Tong, Y., Lapointe, F., Thämer, M., Wolf, M. \& Campen, R. K. Hydrophobic water probed experimentally at the gold electrode/aqueous interface. Angew. Chem. Int. Ed. 56, 4211-4214 (2017).

176. Michaelides, A. Density functional theory simulations of water-metal interfaces: waltzing waters, a novel 2D ice phase, and more. Appl. Phys. A Mater. Sci. Process. 85, 415-425 (2006).

177. Roudgar, A. \& Gro $\beta$, A. Water bilayer on the $\mathrm{Pd} / \mathrm{Au}\left(\begin{array}{lll}1 & 1 & 1\end{array}\right)$ overlayer system: coadsorption and electric field effects. Chem. Phys. Lett. 409, 157-162 (2005).
178. Schlaich, A., Dos Santos, A. P. \& Netz, R. R. Simulations of nanoseparated charged surfaces reveal charge-induced water reorientation and nonadditivity of hydration and mean-field electrostatic repulsion. Langmuir 35, 551-560 (2019).

179. Willard, A. P., Reed, S. K., Madden, P. A. \& Chandler, D. Water at an electrochemical interface - a simulation study. Faraday Discuss. 141, 423-441 (2008).

180. Fayer, M. D. Dynamics of water interacting with interfaces, molecules, and ions. Acc. Chem. Res. 45, 3-14 (2012).

181. Costard, R., Greve, C., Heisler, I. A. \& Elsaesser, T. Ultrafast energy redistribution in local hydration shells of phospholipids: a two-dimensional infrared study. J. Phys. Chem. Lett. 3, 3646-3651 (2012).

182. Nihonyanagi, S., Yamaguchi, S. \& Tahara, T. Ultrafast dynamics at water interfaces studied by vibrational sum frequency generation spectroscopy. Chem. Rev. 117, 10665-10693 (2017)

183. Cyran, J. D., Backus, E. H. G., Nagata, Y. \& Bonn, M. Structure from dynamics: vibrational dynamics of interfacial water as a probe of aqueous heterogeneity. J. Phys. Chem. B 122, 3667-3679 (2018).

184. Deiseroth, M., Bonn, M. \& Backus, E. H. G. Orientation independent vibrational dynamics of lipidbound interfacial water. Phys. Chem. Chem. Phys. 22, 10142-10148 (2020).

185. Eftekhari-Bafrooei, A. \& Borguet, E. Effect of surface charge on the vibrational dynamics of interfacial water. J. Am. Chem. Soc. 131, 12034-12035 (2009).

186. Hsieh, C. S. et al. Ultrafast reorientation of dangling $\mathrm{OH}$ groups at the air-water interface using femtosecond vibrational spectroscopy. Phys. Rev. Lett. 107, 116102 (2011)

187. Xiao, S., Figge, F., Stirnemann, G., Laage, D. \& McGuire, J. A. Orientational dynamics of water at an extended hydrophobic interface. J. Am. Chem. Soc. 138, 5551-5560 (2016)

188. Cheng, L., Fenter, P., Nagy, K. L., Schlegel, M. L. \& Sturchio, N. C. Molecular-scale density oscillations in water adjacent to a mica surface. Phys. Rev. Lett. 87 156103 (2001).

189. Higgins, M. J. et al. Structured water layers adjacent to biological membranes. Biophys. J. 91, 2532-2542 (2006).

190. Fukuma, T \& Garcia, R. Atomic- and molecularresolution mapping of solid-liquid interfaces by 3D atomic force microscopy. ACS Nano 12 , 11785-11797 (2018).

191. Imada, H., Kimura, K. \& Onishi, H. Water and 2-propanol structured on calcite (104) probed by frequency-modulation atomic force microscopy. Langmuir 29, 10744-10751 (2013).

192. Lardge, J. S., Duffy, D. M. \& Gillan, M. J. Investigation of the interaction of water with the calcite (10.4) surface using ab initio simulation. J. Phys. Chem. C 113, 7207-7212 (2009).

193. Zachariah, Z., Espinosa-Marzal, R. M., Spencer, N. D. $\&$ Heuberger, M. P. Stepwise collapse of highly overlapping electrical double layers. Phys. Chem. Chem. Phys. 18, 24417-24427 (2016).

194. Israelachvili, J. N. \& Pashley, R. M. Molecular layering of water at surfaces and origin of repulsive hydration forces. Nature 306, 249-250 (1983).

195. Fukuma, T., Ueda, Y., Yoshioka, S. \& Asakawa, H. Atomic-scale distribution of water molecules at the mica-water interface visualized by three-dimensional scanning force microscopy. Phys. Rev. Lett. 104, 016101 (2010).

196. Bonthuis, D. J., Gekle, S. \& Netz, R. R. Dielectric profile of interfacial water and its effect on doublelayer capacitance. Phys. Rev. Lett. 107, 166102 (2011).

197. Loche, P., Wolde-Kidan, A., Schlaich, A., Bonthuis, D. J. $\S$ Netz, R. R. Comment on "Hydrophobic surface enhances electrostatic interaction in water". Phys. Rev. Lett. 123, 49601 (2019).

198. Loche, P., Ayaz, C., Wolde-kidan, A., Schlaich, A. \& Netz, R. R. Universal and nonuniversal aspects of electrostatics in aqueous nanoconfinement. J. Phys. Chem. B 124, 4365-4371 (2020).

199. Siepmann, J. I. \& Sprik, M. Influence of surface topology and electrostatic potential on water/ electrode systems. J. Chem. Phys. 102, 511-524 (1995).

200. Zhang, C. \& Sprik, M. Computing the dielectric constant of liquid water at constant dielectric displacement. Phys. Rev. B 93, 144201 (2016).

201. Zhang, C. \& Sprik, M. Finite field methods for the supercell modeling of charged insulator/electrolyte interfaces. Phys. Rev. B 94, 245309 (2016). 
202. Otani, M et al. Electrode dynamics from first principles. J. Phys. Soc. Jpn. 77, 024802 (2008).

203. Bouzid, A. \& Pasquarello, A. Atomic-scale simulation of electrochemical processes at electrode/water interfaces under referenced bias potential. J. Phys. Chem. Lett. 9, 1880-1884 (2018).

204. Huang, D. M., Cottin-Bizonne, C., Ybert, C. \& Bocquet, L. Ion-specific anomalous electrokinetic effects in hydrophobic nanochannels. Phys. Rev. Lett. 98, 177801 (2007)

205. Jardat, M., Dufrêche, J. F., Marry, V., Rotenberg, B. $\&$ Tura, P. Salt exclusion in charged porous media: a coarse-graining strategy in the case of montmorillonite clays. Phys. Chem. Chem. Phys. 11, 2023-2033 (2009).

206. Paillusson, F. \& Blossey, R. Slits, plates, and PoissonBoltzmann theory in a local formulation of nonlocal electrostatics. Phys. Rev. E Stat. Nonlin. Soft Matter Phys. 82, 052501 (2010).

207. Ben-Yaakov, D., Andelman, D., Podgornik, R. \& Harries, D. lon-specific hydration effects: extending the Poisson-Boltzmann theory. Curr. Opin. Colloid Interface Sci. 16, 542-550 (2011).

208. Hartkamp, R., Siboulet, B., Dufrêche, J. F. \& Coasne, B. lon-specific adsorption and electroosmosis in charged amorphous porous silica. Phys. Chem. Chem. Phys. 17, 24683-24695 (2015).

209. Nørskov, J. K. et al. Origin of the overpotential for oxygen reduction at a fuel-cell cathode. J. Phys. Chem. B 108, 17886-17892 (2004)

210. Karlberg, G. S. Adsorption trends for water, hydroxyl, oxygen, and hydrogen on transition-metal and platinum-skin surfaces. Phys. Rev. B Condens. Matter Mater. Phys. 74, 153414 (2006)

211. Abild-Pedersen, F. et al. Scaling properties of adsorption energies for hydrogen-containing molecules on transition-metal surfaces. Phys. Rev. Lett. 99, 016105 (2007).

212. Ferrin, P., Kandoi, S., Nilekar, A. U. \& Mavrikakis, M. Hydrogen adsorption, absorption and diffusion on and in transition metal surfaces: a DFT study. Surf. SCi. 606, 679-689 (2012).

213. Serrano, G. et al. Molecular ordering at the interface between liquid water and rutile $\mathrm{TiO}_{2}(110)$. Adv. Mater Interfaces 2, 1500246 (2015).

214. Diebold, U. The surface science of titanium dioxide. Surf. Sci. Rep. 48, 53-229 (2002).

215. Ketteler, G. et al. The nature of water nucleation sites on $\mathrm{TiO}_{2}(110)$ surfaces revealed by ambient pressure X-ray photoelectron spectroscopy. J. Phys. Chem. C $111,8278-8282$ (2007).

216. Zhang, Z. et al. Ion adsorption at the rutile-water interface: linking molecular and macroscopic properties. Langmuir 20, 4954-4969 (2004).

217. Benkoula, S. et al. Water adsorption on $\mathrm{TiO}_{2}$ surfaces probed by soft X-ray spectroscopies: bulk materials vs. isolated nanoparticles. Sci. Rep. 5, 15088 (2015)

218. Chen, J., Li, Y. F., Sit, P. \& Selloni, A. Chemical dynamics of the first proton-coupled electron transfer of water oxidation on $\mathrm{TiO}_{2}$ anatase. J. Am. Chem. Soc. 135, 18774-18777 (2013)

219. Wang, Z. T. et al. Probing equilibrium of molecular and deprotonated water on $\mathrm{TiO}_{2}(110)$. Proc. Natl Acad. Sci. USA 114, 1801-1805 (2017).

220. Diebold, U. Perspective: A controversial benchmark system for water-oxide interfaces: $\mathrm{H}_{2} \mathrm{O} / \mathrm{TiO}_{2}(110)$ J. Chem. Phys. 147, 040901 (2017).

221. Cheng, J. \& Sprik, M. Acidity of the aqueous rutile $\mathrm{TiO}_{2}(110)$ surface from density functional theory based molecular dynamics. J. Chem. Theory Comput. 6 , 880-889 (2010)

222. Cheng, J. \& Sprik, M. The electric double layer at a rutile $\mathrm{TiO}_{2}$ water interface modelled using density functional theory based molecular dynamics simulation. J. Phys. Condens. Matter 26, 244108 (2014).

223. Stamenkovic, V. R. et al. Improved oxygen reduction activity on $\mathrm{Pt}_{3} \mathrm{Ni}(111)$ via increased surface site availability. Science 315, 493-497 (2007).

224. Calle-Vallejo, F., Loffreda, D., Koper, M. T. M. $\delta$ Sautet, P. Introducing structural sensitivity into adsorption-energy scaling relations by means of coordination numbers. Nat. Chem. 7, 403-410 (2015).

225. Calle-Vallejo, F. et al. Finding optimal surface sites on heterogeneous catalysts by counting nearest neighbors. Science 350, 185-189 (2015).

226. Kolb, M. J., Calle-Vallejo, F., Juurlink, L. B. F. \& Koper, M. T. M. Density functional theory study of adsorption of $\mathrm{H}_{2} \mathrm{O}, \mathrm{H}, \mathrm{O}$, and $\mathrm{OH}$ on stepped platinum surfaces. J. Chem. Phys. 140, 134708 (2014).

227. McCrum, I. T. \& Janik, M. J. First principles simulations of cyclic voltammograms on stepped
$\mathrm{Pt}(553)$ and Pt(533) electrode surfaces. ChemElectroChem 3, 1609-1617 (2016).

228. Chen, X., McCrum, I. T., Schwarz, K. A., Janik, M. J. \& Koper, M. T. M. Co-adsorption of cations as the cause of the apparent $\mathrm{pH}$ dependence of hydrogen adsorption on a stepped platinum single-crystal electrode. Angew. Chem. Int. Ed. 56, 15025-15029 (2017).

229. Schiros, T. et al. Structure and bonding of the waterhydroxyl mixed phase on Pt(111). J. Phys. Chem. C 111, 15003-15012 (2007)

230. Lew, W., Crowe, M. C., Campbell, C. T., Carrasco, J. \& Michaelides, A. The energy of hydroxyl coadsorbed with water on Pt(111). J. Phys. Chem. C 115, 23008-23012 (2011).

231. McCrum, I. T. \& Janik, M. J. pH and alkali cation effects on the Pt cyclic voltammogram explained using density functional theory. J. Phys. Chem. C 120 , 457-471 (2016)

232. McCrum, I. T., Chen, X., Schwarz, K. A., Janik, M. J. \& Koper, M. T. M. Effect of step density and orientation on the apparent $\mathrm{pH}$ dependence of hydrogen and hydroxide adsorption on stepped platinum surfaces. J. Phys. Chem. C 122, 16756-16764 (2018).

233. Sheng, W., Gasteiger, H. A. \& Shao-Horn, Y. Hydrogen oxidation and evolution reaction kinetics on platinum acid vs alkaline electrolytes. J. Electrochem. Soc. 157 , 1529-1536 (2010).

234. Durst, J. et al. New insights into the electrochemical hydrogen oxidation and evolution reaction mechanism. Energy Environ. Sci. 7, 2255-2260 (2014).

235. Zheng, J., Sheng, W., Zhuang, Z., Xu, B. \& Yan, Y. Universal dependence of hydrogen oxidation and evolution reaction activity of platinum-group metals on $\mathrm{pH}$ and hydrogen binding energy. Sci. Adv. 2 . e1501602 (2016)

236. Subbaraman, R. et al. Trends in activity for the water electrolyser reactions on $3 d \mathrm{M}(\mathrm{Ni}, \mathrm{Co}, \mathrm{Fe}, \mathrm{Mn})$ hydr(oxy) oxide catalysts. Nat. Mater. 11, 550-557 (2012)

237. Schouten, K. J. P., Van Der Niet, M. J. T. C. \& Koper, M. T. M. Impedance spectroscopy of $\mathrm{H}$ and $\mathrm{OH}$ adsorption on stepped single-crystal platinum electrodes in alkaline and acidic media. Phys. Chem. Chem. Phys. 12, 15217-15224 (2010)

238. Mamatkulov, S. I., Rinne, K. F., Buchner, R., Netz, R. R. $\&$ Bonthuis, D. J. Water-separated ion pairs cause the slow dielectric mode of magnesium sulfate solutions. J. Chem. Phys. 148, 222812 (2018).

239. Garg, M. \& Kern, K. Attosecond coheren manipulation of electrons in tunneling microscopy. Science 367, 411-415 (2020)

240. Tong, Y., Lapointe, F., Wolf, M. \& Kramer Campen, M. Probing the birth and ultrafast dynamics of hydrated electrons at the gold/liquid water interface via an optoelectronic approach. J. Am. Chem. Soc. 142 18619-18627 (2020).

241. Söngen, H. et al. The weight function for charges - A rigorous theoretical concept for Kelvin probe force microscopy. J. Appl. Phys. 119, 025304 (2016).

242. Watkins, M. \& Reischl, B. A simple approximation for forces exerted on an AFM tip in liquid. J. Chem. Phys. 138,154703 (2013)

243. Amano, K. I., Suzuki, K., Fukuma, T., Takahashi, O. $\&$ Onishi, $\mathrm{H}$. The relationship between local liquid density and force applied on a tip of atomic force microscope: a theoretical analysis for simple liquids. J. Chem Phys. 139, 224710 (2013).

244. Söngen, $H$. et al. Chemical identification at the solidliquid interface. Langmuir 33, 125-129 (2017).

245. Söngen, H., Nalbach, M., Adam, H. \& Kühnle, A. Three-dimensional atomic force microscopy mapping at the solid-liquid interface with fast and flexible data acquisition. Rev. Sci. Instrum. 87, 063704 (2016).

246. Lyklema, J. Interfacial potentials: measuring the immeasurable? Substantia 1, 75-93 (2017).

247. Lyklema, J. Fundamentals of Interface and Colloid Science: Solid-Liquid Interfaces (Elsevier, 1995).

248. Taylor, D. M., De Oliveira, O. N. \& Morgan, H. Models for interpreting surface potential measurements and their application to phospholipid monolayers. J. Colloid Interface Sci. 139, 508-518 (1990).

249. Brockman, H. Dipole potential of lipid membranes. Chem. Phys. Lipids 73, 57-79 (1994).

250. Moncelli, M. R., Becucci, L., Buoninsegni, F. T. \& Guidelli, R. Surface dipole potential at the interface between water and self-assembled monolayers of phosphatidylserine and phosphatidic acid. Biophys. $J$. 74, 2388-2397 (1998)

251. Wang, L. Measurements and implications of the membrane dipole potential. Annu. Rev. Biochem. 81, 615-635 (2012).
252. Casper, C. B., Verreault, D., Adams, E. M., Hua, W. \& Allen, H. C. Surface potential of DPPC monolayers on concentrated aqueous salt solutions. J. Phys. Chem. B 120, 2043-2052 (2016)

253. Paltauf, F., Hauser, H. \& Phillips, M. C. Monolayer characteristics of some 1,2-diacyl, 1-alkyl-2-acyl and 1, 2-dialkyl phospholipids at the air-water interface. Biochim. Biophys. Acta Biomembr. 249, 539-547 (1971).

254. Dreier, L. B., Bernhard, C., Gonella, G., Backus, E. H. G. \& Bonn, M. Surface potential of a planar charged lipid-water interface. What do vibrating plate methods, second harmonic and sum frequency measure? J. Phys. Chem. Lett. 9, 5685-5691 (2018).

255. Nonnenmacher, M., O’Boyle, M. P. \& Wickramasinghe, H. K. Kelvin probe force microscopy. Appl. Phys. Lett. 58, 2921-2923 (1991).

256. Melitz, W., Shen, J., Kummel, A. C. \& Lee, S. Kelvin probe force microscopy and its application. Surf. Sci. Rep. 66, 1-27 (2011).

257. Neff, G. A., Helfrich, M. R., Clifton, M. C. \& Page, C. J. Layer-by-layer growth of acentric multilayers of $\mathrm{Zr}$ and azobenzene bis(phosphonate): structure, composition, and second-order nonlinear optical properties. Chem. Mater. 12, 2363-2371 (2000).

258. Rahe, P. \& Söngen, H. in Kelvin Probe Force Microscopy: From Single Charge Detection to Device Characterization (eds Sadewasser, S. \& Glatzel, T.) 147-170 (Springer, 2018).

259. Kobayashi, N., Asakawa, H. \& Fukuma, T. Nanoscale potential measurements in liquid by frequency modulation atomic force microscopy. Rev. Sci. Instrum. 81, 123705 (2010).

260. Hüfner, S. Photoelectron Spectroscopy: Principles and Applications (Springer, 1996).

261. Seidel, R., Thürmer, S. \& Winter, B. Photoelectron spectroscopy meets aqueous solution: studies from a vacuum liquid microjet. J. Phys. Chem. Lett. $\mathbf{2}$, 633-641 (2011)

262. Winter, B. Liquid microjet for photoelectron spectroscopy. Nucl. Instrum. Methods Phys. Res. A Accel. Spectrom. Detect. Assoc. Equip. 601 139-150 (2009).

263. Kolmakov, A. et al. Graphene oxide windows for in situ environmental cell photoelectron spectroscopy. Nat. Nanotechnol. 6, 651-657 (2011).

264. Kraus, J. et al. Photoelectron spectroscopy of wet and gaseous samples through graphene membranes. Nanoscale 6, 14394-14403 (2014)

265. Petit, T. et al. X-ray absorption spectroscopy of $\mathrm{TiO}_{2}$ nanoparticles in water using a holey membrane-based flow cell. Adv. Mater. Interfaces 4, 1700755 (2017).

266. de Beer, A. G. F., Campen, R. K. \& Roke, S. Separating surface structure and surface charge with secondharmonic and sum-frequency scattering. Phys. Rev. B 82, 235431 (2010).

267. Gonella, G., Luetgebaucks, C., de Beer, A. G. F. $\delta$ Roke, S. Second harmonic and sum-frequency generation from aqueous interfaces is modulated by interference. J. Phys. Chem. C 120, 9165-9173 (2016).

268. Ohno, P. E., Saslow, S. A., Wang, H., Geiger, F. M. $\&$ Eisenthal, K. B. Phase-referenced nonlinear spectroscopy of the $\alpha$-quartz/water interface. Nat. Commun. 7, 13587 (2016)

269. Lee, C. H., Chang, R. K. \& Bloembergen, N. Nonlinear electroreflectance in silicon and silver. Phys. Rev. Lett. 18, 167-170 (1967)

270. Geiger, F. M. Second harmonic generation, sum frequency generation, and $\chi^{(3)}$ : dissecting environmental interfaces with a nonlinear optical Swiss Army knife. Annu. Rev. Phys. Chem. 60, 61-83 (2009).

271. Achtyl, J. L. et al. Free energy relationships in the electrical double layer over single-layer graphene. J. Am. Chem. Soc. 135, 979-981 (2013).

272. Troiano, J. M. et al. Quantifying the electrostatics of polycation-lipid bilayer interactions. J. Am. Chem. Soc. 139, 5808-5816 (2017).

273. Mcgeachy, A. C. et al. Counting charges on membranebound peptides. Chem. Sci. 9, 4285-4298 (2018).

274. Yan, E. C. Y., Liu, Y. \& Eisenthal, K. B. New method for determination of surface potential of microscopic particles by second harmonic generation. J. Phys. Chem. B 102, 6331-6336 (1998)

275. Marchioro, A. et al. Surface characterization of colloidal silica nanoparticles by second harmonic scattering: quantifying the surface potential and interfacial water order. J. Phys. Chem. C 123 , 20393-20404 (2019).

276. Didier, M. E. P., Tarun, O. B., Jourdain, P., Magistretti, P. \& Roke, S. Membrane water for probing neuronal 
membrane potentials and ionic fluxes at the single cell level. Nat. Commun. 9, 5287 (2018).

277. Israelachvili, J. et al. Recent advances in the surface forces apparatus (SFA) technique. Rep. Prog. Phys. 73, 036601 (2010)

278. Magnussen, O. M. Atomic-scale insights into electrode surface dynamics by high-speed scanning probe microscopy. Chem. Eur. J. 25, 12865-12883 (2019).

279. Fukuma, T., Onishi, K., Kobayashi, N., Matsuki, A. \& Asakawa, $\mathrm{H}$. Atomic-resolution imaging in liquid by frequency modulation atomic force microscopy using small cantilevers with megahertz-order resonance frequencies. Nanotechnology 23, 135706 (2012).

280. Signorell, R. Electron scattering in liquid water and amorphous ice: a striking resemblance. Phys. Rev. Lett. 124, 205501 (2020)

281. Jahnke, T. et al. Interatomic and intermolecular coulombic decay. Chem. Rev. 120, 11295-11369 (2020).

282. Seidel, R., Winter, B. \& Bradforth, S. E. Valence electronic structure of aqueous solutions: insights from photoelectron spectroscopy. Annu. Rev. Phys. Chem. 67, 283-305 (2016).

283. Golnak, R. et al. Joint analysis of radiative and nonradiative electronic relaxation upon X-ray irradiation of transition metal aqueous solutions. Sci. Rep. 6 , 24659 (2016).
284. Winter, B. Interfaces: scientists strike wet gold. Nat. Chem. 7, 192-194 (2015).

285. Shen, Y. R. The Principles of Nonlinear Optics (Wiley, 1984).

286. Boyd, R. W. Nonlinear Optics (Academic Press/ Elsevier, 2008)

287. Morita, A. Theory of Sum Frequency Generation Spectroscopy (Springer, 2018).

288. Jena, K. C., Covert, P. A. \& Hore, D. K. The effect of salt on the water structure at a charged solid surface: differentiating second- and third-order nonlinear contributions. J. Phys. Chem Lett. 2, 1056-1061 (2011).

289. Liljeblad, J. F. D., Bulone, V., Rutland, M. W. \& Johnson, C. M. Supported phospholipid monolayers. The molecular structure investigated by vibrational sum frequency spectroscopy. J. Phys. Chem. C 115 10617-10629 (2011).

290. De Beer, A. G. F., Campen, R. K. \& Roke, S. Separating surface structure and surface charge with secondharmonic and sum-frequency scattering. Phys. Rev. B Condens. Matter Mater. Phys 82, 235431 (2010).

291. Campen, R. K., Pymer, A. K., Nihonyanagi, S. \& Borguet, E. Linking surface potential and deprotonation in nanoporous silica: second harmonic generation and acid/base titration. J. Phys. Chem. C 114, 18465-18473 (2010).

\section{Acknowledgements}

The authors thank Katrin F. Domke, Daria Maltseva, Takakatsu Seki and Chun-Chieh Yu for their careful reading and Hagen Sögen for providing Fig. 6d,e. R.R.N. acknowledges support from the Deutsche Forschungsgemeinschaft (DFG) via grant SFB 1078. They are grateful to the MaxWater initiative of the Max Planck Society for support.

\section{Author contributions}

M.B., G.G., Y.N., R.K.C., E.H.G.B. and R.R.N. conceived the manuscript. M.B. and G.G. wrote it together with Y.N., R.K.C., E.H.G.B., R.R.N., A.K., I.T.M., M.T.M.K. and B.W. All authors contributed to the discussions, revisions and editing of the article.

Competing interests

The authors declare no competing interests.

\section{Peer review information}

Nature Reviews Chemistry thanks the anonymous reviewers for their contribution to the peer review of this work.

\section{Publisher's note}

Springer Nature remains neutral with regard to jurisdictional claims in published maps and institutional affiliations.

(C) Springer Nature Limited 2021 